\title{
Gravitational instability of finite isothermal spheres
}

\begin{abstract}
P. H. Chavanis ${ }^{\star}$
Laboratoire de Physique Quantique, Université Paul Sabatier, 118 route de Narbonne, 31062 Toulouse, France Institute for Theoretical Physics, University of California, Santa Barbara, California CA93106, USA

Received 19 March 2001 / Accepted 6 August 2001

Abstract. We investigate the stability of bounded self-gravitating systems in the canonical ensemble by using a thermodynamical approach. Our study extends the earlier work of Padmanabhan (1989) in the microcanonical ensemble. By studying the second variations of the free energy, we find that instability sets in precisely at the point of minimum temperature in agreement with the theorem of Katz (1978). The perturbation that induces instability at this point is calculated explicitly; it has not a "core-halo" structure contrary to what happens in the microcanonical ensemble. We also study Jeans type gravitational instability of isothermal gaseous spheres described by Navier-Stokes equations. The introduction of a container and the consideration of an inhomogeneous distribution of matter avoids the Jeans swindle. We show analytically the equivalence between dynamical stability and thermodynamical stability and the fact that the stability of isothermal gas spheres does not depend on the viscosity. This confirms the findings of Semelin et al. (2001) who used numerical methods or approximations. We also give a simpler derivation of the geometric hierarchy of scales inducing instability discovered by these authors. The density profiles that trigger these instabilities are calculated explicitly; high order modes of instability present numerous oscillations whose nodes also follow a geometric progression. This suggests that the system will fragment in a series of "clumps" and that these "clumps" will themselves fragment in substructures. The fact that both the domain sizes leading to instability and the "clumps" sizes within a domain follow a geometric progression with the same ratio suggests a fractal-like behavior. This gives further support to the interpretation of de Vega et al. (1996) concerning the fractal structure of the interstellar medium.
\end{abstract}

Key words. hydrodynamics - instabilities

\section{Introduction}

The thermodynamics of self-gravitating systems is a fascinating subject. It started with Antonov (1962)'s discovery that, when a self-gravitating system is confined within a box of radius $R$, no maximum entropy state can exist below a certain critical energy $E=-0.335 G M^{2} / R$. This intriguing result was further discussed by LyndenBell \& Wood (1968) who conjectured that for $E<$ $-0.335 G M^{2} / R$ the system would collapse and overheat. This is called "gravothermal catastrophe" or "Antonov instability". Lynden-Bell \& Wood have related this phenomenon to the very particular property of self-gravitating systems to possess negative specific heats. The gravothermal catastrophe picture has been confirmed by sophisticated numerical simulations (Larson 1970; Cohn 1980; Lynden-Bell \& Eggleton 1980) and is expected to play a crucial role in the evolution of globular clusters. It is found that the collapse proceeds self-similarly (with power law behaviors) and that the central density becomes infinite in a finite time. This instability has been known as "core

\footnotetext{
* e-mail: chavanis@irsamc2.ups-tlse.fr
}

collapse" and many globular clusters have probably experienced core collapse (Binney \& Tremaine 1987). In the case of dense clusters of compact stars (neutron stars or stellar mass black holes), the gravothermal catastrophe can lead to the formation of supermassive black holes of the right size to explain quasars and AGNs (Shapiro \& Teukolsky 1995). Statistical mechanics is also relevant for collisionless self-gravitating systems (e.g., elliptical galaxies, dark matter, ...) undergoing a violent relaxation (Lynden-Bell 1967; Chavanis et al. 1996; Chavanis \& Sommeria 1998; Chavanis 1998a, 2001a). In particular, the inner regions of elliptical galaxies are close to isothermal and this is an important ingredient to understand de Vaucouleurs' $R^{1 / 4}$ law (Hjorth \& Madsen 1993).

On a theoretical point of view, the stability of isothermal spheres has been first investigated by Katz (1978) with a very powerful method extending Poincaré's theory of linear series of equilibrium. He found that instability sets in precisely at the point of minimum energy. This stability analysis was reconsidered by Padmanabhan (1989) who studied the sign of the second variations of entropy and reduced the problem of stability to an eigenvalue equation. This leads to the same stability limit as 
Katz but the method of Padmanabhan provides in addition the form of the perturbation that induces instability at the critical point. It is found that this perturbation presents a "core-halo" structure.

The analysis of Padmanabhan (1989) was performed in the microcanonical ensemble in which the energy is fixed. The microcanonical ensemble is probably the most relevant for studying stellar systems like elliptical galaxies or globular clusters (Binney \& Tremaine 1987). Indeed, apart from a slow evaporation, these systems can be assumed isolated so the evolution conserves energy $E$ and mass $M$. In addition, from the viewpoint of statistical mechanics, only the microcanonical ensemble is rigorously justified for non extensive systems, as discussed in the review of Padmanabhan (1990). However, it is always possible to define formally a canonical ensemble or a grand canonical ensemble in which the temperature is fixed instead of the energy. As suggested by de Vega et al. (1996a, 1996b) these ensembles may be suitable for describing the cold interstellar medium where the temperature is imposed by the cosmic background radiation at $T \sim 3 \mathrm{~K}$ in the outer parts of galaxies, devoid of any star and heating sources (Pfenninger et al. 1994; Pfenninger \& Combes 1994). In particular, by working out the statistical mechanics of the self-gravitating gas, de Vega et al. (1996a, 1996b) have shown that self-gravity can provide a dynamical mechanism to produce the fractal structure of the interstellar medium. They used the same approach to explain the fractal structure of the universe (de Vega et al. 1998), assuming that galaxies have reached a quasi-thermodynamical equilibrium like in the early work of Saslaw \& Hamilton (1984).

For self-gravitating systems, it is well known that the thermodynamical ensembles do not coincide in the whole range of parameters (Padmanabhan 1990). Using toy models, Lynden-Bell \& Lynden-Bell (1977) and Padmanabhan (1990) demonstrated that the region of negative specific heats allowed in the microcanonical ensemble is replaced by a phase transition in the canonical ensemble. This phase transition separates a dilute "gaseous" phase from a dense "collapsed" phase. Since these toy models are not very realistic, the self-gravitating gas was also studied in a meanfield approximation. In this viewpoint, an isothermal sphere is stable if and only if it is a local maximum of an appropriate thermodynamical potential (the entropy in the microcanonical ensemble and the free energy in the canonical ensemble). As expected from physical grounds, phase transition occurs when the gaseous sphere ceases to be a local maximum of this potential and becomes a saddle point. In this paper, we investigate the stability of isothermal gaseous spheres in the canonical ensemble by studying the sign of the second variations of the free energy. Our analysis is a direct extension of Padmanabhan (1989)'s approach in the microcanonical ensemble. The two studies therefore provide a unified description of the stability of isothermal spheres in the meanfield approximation in terms of thermodynamical potentials.
For a long time, the thermodynamics of self-gravitating systems was considered exclusively in a meanfield approach (or with toy models). However, recently, de Vega \& Sanchez (2001a,b) have developed a rigorous statistical mechanics of self-gravitating systems by using field theoretical methods. In particular, they evidenced the existence of a thermodynamic limit in which the number of particles $N$ and the volume $R^{3}$ go to infinity keeping $N / R$ fixed. This very unusual thermodynamic limit proves to be appropriate to non extensive systems. By using Monte Carlo simulations and analytical calculations, they showed that the meanfield approximation correctly describes the thermodynamic limit except near the critical points where a phase transition occurs. They also derived the local equation of state of a self-gravitating gas instead of assuming it, as is done in the meanfield treatments. Therefore, their work fully justifies the studies of previous authors and specifies their range of validity.

This paper is organized as follows. In Sect. 2, we introduce a meanfield description of the system in the canonical ensemble and show that critical points of free energy $J$ at fixed temperature $T$ and mass $M$ correspond to isothermal spheres like those studied in the context of stellar structure (Chandraskhar 1942). We show that there is no global maximum of free energy. There is not even a local maximum of free energy in an unbounded domain: unbounded isothermal spheres have an infinite mass! We restrict therefore our analysis to the case of self-gravitating systems confined within a spherical box of radius $R$. In this case, there exists local maxima of $J$ (metastable states) if the normalized temperature $\eta=\frac{\beta G M}{R}$ is less than 2.52 and the density contrast $\mathcal{R}=\rho(0) / \rho(R)<32.1$. Critical points of free energy with density contrast $\mathcal{R}>32.1$ are unstable saddle points. For $\eta>2.52$, there are not even critical points of free energy: in that case, the system is expected to undergo a phase transition and collapse. This "isothermal collapse" is the counterpart of the "gravothermal catastrophe" in the microcanonical ensemble (see Figs. 2, 3).

In Sect. 3, we study the sign of the second variations of free energy by using the methods of Padmanabhan (1989) introduced in the microcanonical ensemble. We show analytically that instability sets in precisely at the point of minimum temperature in agreement with the theorem of Katz (1978). The perturbation that induces instability at this point is calculated explicitly; it has not a "core-halo" structure contrary to what happens in the microcanonical ensemble.

In Sect. 4, we study Jeans type gravitational instability of isothermal gaseous spheres described by NavierStokes equations. The introduction of a container removes the problems associated with an infinite homogeneous medium and avoids the Jeans swindle. We show analytically the equivalence between dynamical stability and thermodynamical stability and the fact that the stability of isothermal gas spheres does not depend on the viscosity. This confirms the findings of Semelin et al. (2001) who used numerical methods or approximations. We also give 
a simpler derivation of the geometric hierarchy of scales inducing instability discovered by these authors using sophisticated renormalization group technics (Semelin et al. 1999). This provides a more illuminating interpretation of their results.

In Sect. 5, we make speculations about the fragmentation and the fractal structure of an isothermal selfgravitating gas. We distinguish between the Jeans length $L_{\mathrm{J}}$ defined with the mean density and the King's length $L_{\mathrm{K}}$ defined with the central density. If we fix the Jeans length, instability occurs for $R>\sqrt{\frac{2.52 \ldots}{3}} L_{\mathrm{J}}$ and is marked by the absence of critical point of free energy (i.e., hydrostatic equilibrium) above this threshhold. In that case the system is expected to collapse without fragmenting. If we fix the King's length (or core size), a first instability occurs for $R=\frac{8.99 \ldots}{3} L_{\mathrm{K}}$. Above this threshold critical points of free energy still exist but they are unstable saddle points. Secondary instabilities occur at larger box radii that asymptotically follow a geometric progression $R_{n} \sim[10.74 \ldots]^{n} L_{\mathrm{K}}$. The density profiles that trigger these high order modes of instability are calculated explicitly. They present more and more oscillations whose nodes also follow a geometric progression $r_{n} \sim[10.74 \ldots]^{n} R$. The profile that destabilizes the singular isothermal sphere has an infinite number of nodes! Each oscillation can be interpreted as a "germ" in the langage of phase transition and the above picture suggests that the system will fragment into a series of "clumps". It is expected that these "clumps" will evolve by achieving higher and higher density contrasts, and finally fragment in turn into substructures. This yields a hierarchy of structures fitting one into each other in a self-similar way. This picture is given further support by the fact that both the domain sizes inducing instability and the zeros of the perturbation profile in each domain follow a geometric progression with the same ratio. This double-geometric progression may explain in a natural way the fractal structure of a self-gravitating gas like the interstellar medium and the large scale structures of the universe. This gives further support to the interpretation of de Vega et al. (1996a, 1996b, 1998) who emphasized the importance played by self-gravity in building a fractal distribution of matter.

\section{Thermodynamical stability of self-gravitating systems in the canonical ensemble}

\subsection{The free energy}

Consider a system of $N$ particles, each of mass $m$, interacting via Newtonian gravity. The particles can be galaxies, stars, atoms, etc. We assume that the system is non rotating and non expanding. Let $f(\boldsymbol{r}, \boldsymbol{v}, t)$ denote the distribution function of the system, i.e. $f(\boldsymbol{r}, \boldsymbol{v}, t) \mathrm{d}^{3} \boldsymbol{r} \mathrm{d}^{3} \boldsymbol{v}$ gives the mass of particles whose position and velocity are in the cell $\left(\boldsymbol{r}, \boldsymbol{v} ; \boldsymbol{r}+\mathrm{d}^{3} \boldsymbol{r}, \boldsymbol{v}+\mathrm{d}^{3} \boldsymbol{v}\right)$ at time $t$. The integral of $f$ over the velocity determines the spatial density

$\rho=\int f \mathrm{~d}^{3} \boldsymbol{v}$

(1)
On the other hand, in the meanfield approximation, the total mass and the total energy of the system can be expressed as

$$
\begin{aligned}
& M=N m=\int \rho \mathrm{d}^{3} \boldsymbol{r}, \\
& E=\frac{1}{2} \int f v^{2} \mathrm{~d}^{3} \boldsymbol{r} \mathrm{d}^{3} \boldsymbol{v}+\frac{1}{2} \int \rho \Phi \mathrm{d}^{3} \boldsymbol{r}=K+W,
\end{aligned}
$$

where $K$ is the kinetic energy and $W$ the potential energy. The gravitational potential $\Phi$ is related to the star density by the Newton-Poisson equation

$\Delta \Phi=4 \pi G \rho$.

Finally, the Boltzmann entropy is given by the standard formula

$S=-k \int \frac{f}{m} \ln \frac{f}{m} \mathrm{~d}^{3} \boldsymbol{r} \mathrm{d}^{3} \boldsymbol{v}$,

which can be obtained by counting the number of microstates corresponding to a given macrostate and taking the logarithm of this number (Ogorodnikov 1965).

We shall work in the canonical ensemble in which the temperature is fixed, allowing the energy to fluctuate. In that case, the relevant thermodynamical potential is the Massieu function related to the Helmholtz free energy $F=$ $E-T S$ by $J=-\frac{1}{T} F$. Hence

$J=S-\frac{1}{T} E$.

At equilibrium, the system is expected to be in a state that maximizes the Massieu function (6) for a fixed total mass $M$ (in the following we shall call $J$ the free energy, although this is only the free energy up to a negative proportionality factor).

\subsection{The isothermal gaseous spheres}

Following Padmanabhan (1989)'s procedure, we start to maximize the free energy $J$ for a given density field $\rho(\boldsymbol{r})$. This yields the Maxwell-Boltzmann distribution

$f=\left(\frac{m}{2 \pi k T}\right)^{3 / 2} \rho(\boldsymbol{r}) \mathrm{e}^{-\frac{m v^{2}}{2 k T}}$,

which is a global maximum of $J$ with the previous constraint. Substituting this optimal distribution function in Eqs. (3)-(5), we can express the energy and the entropy in terms of the spatial density in the form

$E=\frac{3}{2} N k T+\frac{1}{2} \int \rho \Phi \mathrm{d}^{3} \boldsymbol{r}$

$\frac{S}{k}=\frac{3 N}{2}+\frac{3 N}{2} \ln \left(\frac{2 \pi k T}{m}\right)-\int \frac{\rho}{m} \ln \frac{\rho}{m} \mathrm{~d}^{3} \boldsymbol{r}$. 
We can now determine the variations of $J$ around a given density profile $\rho(\boldsymbol{r})$. To second order in the expansion we get

$$
\begin{array}{r}
\delta J=-\frac{k}{m} \int \delta \rho\left(\ln \frac{\rho}{m}+1\right) \mathrm{d}^{3} \boldsymbol{r}-\frac{k}{m} \int \frac{(\delta \rho)^{2}}{2 \rho} \mathrm{d}^{3} \boldsymbol{r} \\
-\frac{1}{T} \int \Phi \delta \rho \mathrm{d}^{3} \boldsymbol{r}-\frac{1}{2 T} \int \delta \rho \delta \Phi \mathrm{d}^{3} \boldsymbol{r} .
\end{array}
$$

Introducing a Lagrange multiplier $\alpha$ to satisfy the conservation of mass, the condition that $J$ is an extremum is written (to first order)

$0=\delta J-\alpha \delta M=-\int \mathrm{d}^{3} \boldsymbol{r}\left[\frac{\Phi}{T}+\frac{k}{m}\left(\ln \frac{\rho}{m}+1\right)+\alpha\right] \delta \rho$.

This condition must be satisfied for any variations $\delta \rho$. This yields the Boltzmann distribution

$\rho=A \mathrm{e}^{-\beta \Phi}$,

where we have set

$\beta=\frac{m}{k T}$.

Therefore, the Boltzmann distribution (12) is a critical point of free energy. This does not insure, however, that it is a maximum of $J$. It is not even clear that the extremum problem leading to Eq. (12) has a solution. Indeed, the gravitational potential that appears in Eq. (12) must be determined self-consistently by solving the mean field equation

$\Delta \Phi=4 \pi G A \mathrm{e}^{-\beta \Phi}$,

obtained by substituting the density (12) in the Poisson Eq. (4), and relating the Lagrange multiplier $A$ to the constraint $M$. As we shall see, this problem does not always have a solution. When a solution exists, we must consider the sign of the second order variations $\delta^{2} J$ to determine whether it is a maximum or not.

\subsection{Absence of global maximum of free energy}

It is easy to show that there is no global maximum of free energy. To prove this result, we just need to consider a homogeneous sphere of mass $M$ and radius $R$ at temperature $T$. The total energy of this sphere is

$E=\frac{3}{2} N k T-\frac{3 G M^{2}}{5 R}$,

and its free energy

$J=\frac{3}{2} N k \ln \left(\frac{2 \pi k T}{m}\right)-N k \ln \left(\frac{3 N}{4 \pi R^{3}}\right)+\frac{3 G M^{2}}{5 T R}$.

From the above formula it is clear that $J$ goes to $+\infty$ when we spread the density to infinity $(R \rightarrow+\infty)$ or when we contract the system to a point $(R \rightarrow 0)$. Since the mass is conserved in this process no global maximum of free energy can exist.
In addition, there is not even a local maximum of free energy in an unbounded domain: unbounded isothermal spheres have an infinite mass. For non rotating systems, the equilibrium states are expected to be spherically symmetric. In that case, the Boltzmann-Poisson Eq. (14) can be rewritten

$\frac{1}{r^{2}} \frac{\mathrm{d}}{\mathrm{d} r}\left(r^{2} \frac{\mathrm{d} \Phi}{\mathrm{d} r}\right)=4 \pi G A \mathrm{e}^{-\beta \Phi}$.

This equation has been studied extensively in the context of isothermal gaseous spheres (Chandrasekhar 1942). We can check by direct substitution that the distribution

$\Phi_{s}(r)=\frac{1}{\beta} \ln \left(2 \pi G \beta A r^{2}\right), \quad \rho_{s}(r)=\frac{1}{2 \pi G \beta r^{2}}$,

is an exact solution of Eq. (17) known as the singular isothermal sphere (Binney \& Tremaine 1987). Since $\rho \sim$ $r^{-2}$ at large distances, the total mass of the system $M=$ $\int_{0}^{+\infty} \rho 4 \pi r^{2} \mathrm{~d} r$ is infinite! More generally, we can show that any solution of the meanfield Eq. (17) behaves like the singular sphere as $r \rightarrow+\infty$ and has therefore an infinite mass (Chandrasekhar 1942).

We shall avoid the infinite mass problem by confining artificially the system within a spherical box of radius $R$. It is only under this simplifying assumption that a rigorous thermodynamics of self-gravitating systems can be carried out. Physically, the maximum cut-off determines the scale at which other processes intervene to limitate the spatial extent of the system. It is clear from Eq. (16) that the introduction of an upper cut-off does not remove the absence of global maximum of free energy (the system can always increase $J$ by collapsing to a point). However, the presence of a wall at radius $R$ allows the existence of local maxima of free energy (metastable states).

\subsection{The equilibrium phase diagram}

The microcanonical and canonical ensembles yield the same critical points, i.e. the critical points of entropy at fixed mass and energy and the critical points of free energy at fixed mass and temperature coincide. Only the onset of instability will differ from one ensemble to the other. The thermodynamical parameters for bounded isothermal spheres in the meanfield approximation have been calculated by Lynden-Bell \& Wood (1968) and we shall directly use their results.

To that purpose, we introduce the function $\psi=\beta(\Phi-$ $\left.\Phi_{0}\right)$ where $\Phi_{0}$ is the gravitational potential at $r=0$. Then, the density field can be written

$\rho=\rho_{0} \mathrm{e}^{-\psi}$,

where $\rho_{0}$ is the central density. Introducing the notation $\xi=\left(4 \pi G \beta \rho_{0}\right)^{1 / 2} r$, the Boltzmann-Poisson Eq. (17) reduces to the standard Emden form (Chandrasekhar 1942)

$\frac{1}{\xi^{2}} \frac{\mathrm{d}}{\mathrm{d} \xi}\left(\xi^{2} \frac{\mathrm{d} \psi}{\mathrm{d} \xi}\right)=\mathrm{e}^{-\psi}$ 


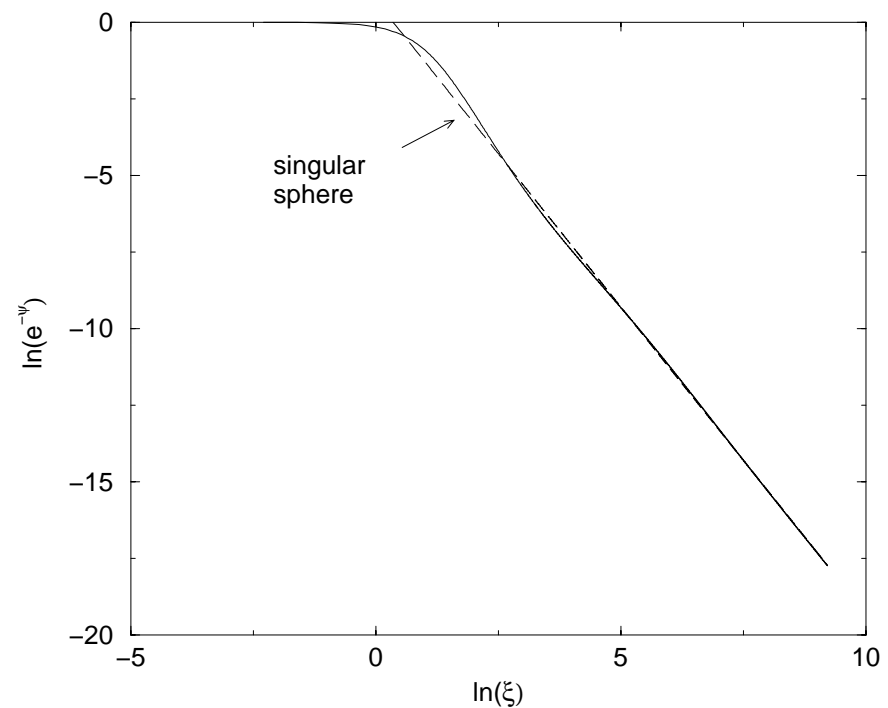

Fig. 1. Normalized density profile of the regular spheres compared with the singular profile (dashed line).

Equation (20) has a simple analytic solution, the singular sphere

$\mathrm{e}^{-\psi_{s}}=\frac{2}{\xi^{2}}$

The regular solution of Eq. (20) satisfying the boundary conditions

$\psi=\psi^{\prime}=0$,

when $\xi=0$, must be computed numerically (see Fig. 1). However, its asymptotic behaviors are well-known

$\psi=\frac{1}{6} \xi^{2}-\frac{1}{120} \xi^{4}+\frac{1}{1890} \xi^{6}+\ldots \quad(\xi \rightarrow 0)$,

$\mathrm{e}^{-\psi}=\frac{2}{\xi^{2}}\left\{1+\frac{A}{\xi^{1 / 2}} \cos \left(\frac{\sqrt{7}}{2} \ln \xi+\delta\right)\right\} \quad(\xi \rightarrow+\infty)$.

The curve (24) intersects the singular solution (21) infinitely often at points that asymptotically increase geometrically in the ratio $1: \mathrm{e}^{2 \pi / \sqrt{7}}=1: 10.74 \ldots$ This property will have important physical consequences in Sect. 5 .

In the case of bounded isothermal systems, the solution of Eq. (20) is terminated by the box at different radii given by $\alpha=\left(4 \pi G \beta \rho_{0}\right)^{1 / 2} R$. Lynden-Bell \& Wood (1968) show that the reduced temperature and reduced energy can be expressed in terms of $\alpha$ by

$\eta \equiv \frac{\beta G M}{R}=\alpha \psi^{\prime}(\alpha)$

$\Lambda \equiv-\frac{E R}{G M^{2}}=\frac{3}{2} \frac{1}{\alpha \psi^{\prime}(\alpha)}-\frac{\mathrm{e}^{-\psi(\alpha)}}{\psi^{\prime}(\alpha)^{2}}$.

For each value of the normalized inverse temperature $\frac{\beta G M}{R}$, we can solve Eq. (25) to get $\alpha$. Substituting the result in Eq. (26), we deduce the corresponding value of the normalized energy $-\frac{E R}{G M^{2}}$. We can then determine

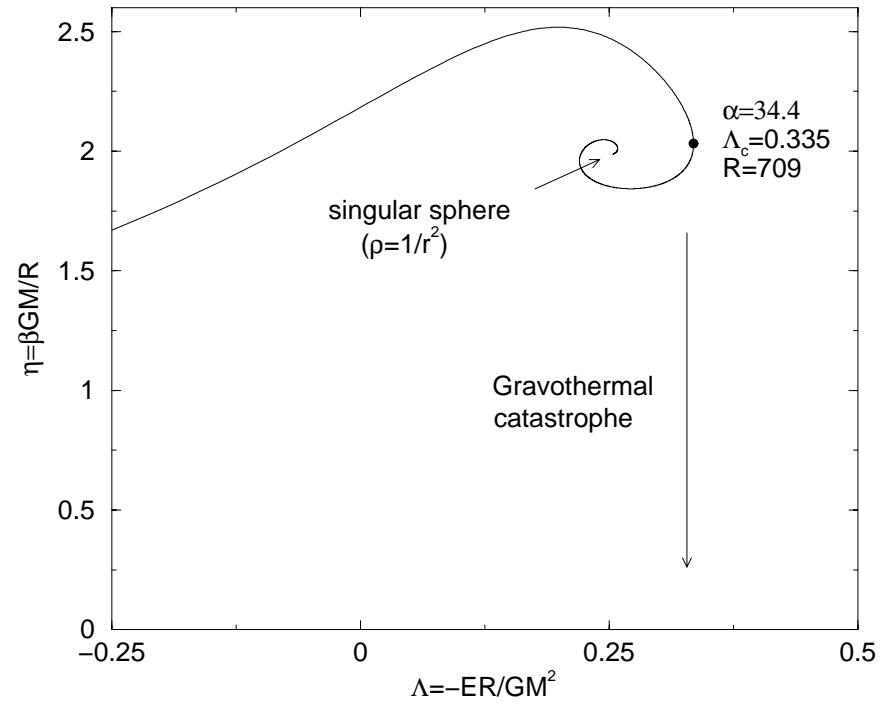

Fig. 2. Stability diagram for isothermal spheres in the microcanonical ensemble (fixed $E$ ).

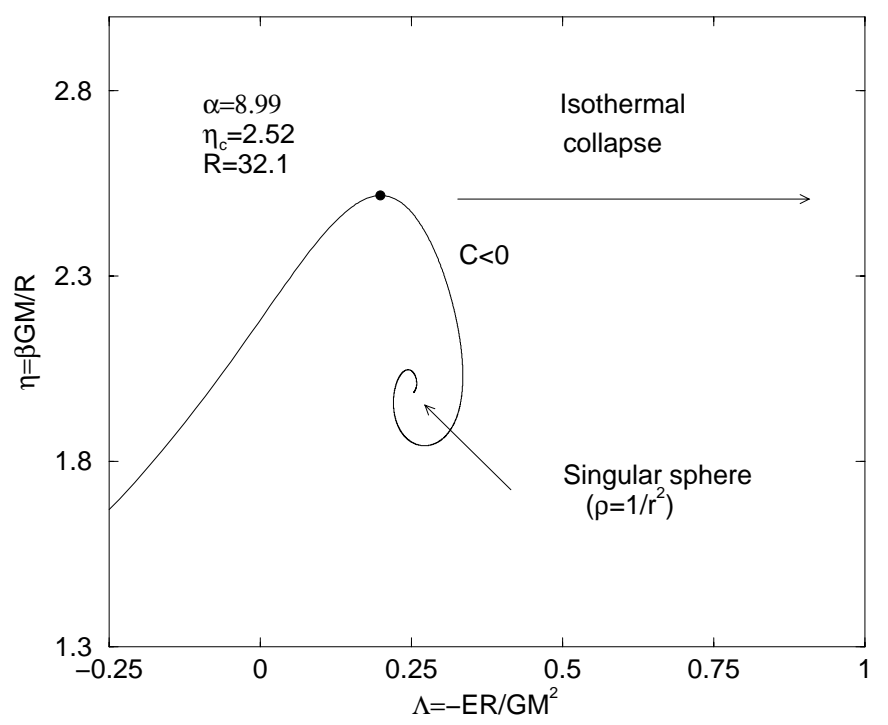

Fig. 3. Stability diagram for isothermal spheres in the canonical ensemble (fixed $T$ ).

the equilibrium diagram $E-\beta$ (Figs. 2, 3). The critical points of entropy or free energy form a spiral. This curve is parametrized by $\alpha$ that goes from 0 to $+\infty$ as we spiral inward. Instead of the parameter $\alpha$, it may be more relevant to introduce the density contrast

$\mathcal{R}=\frac{\rho_{0}}{\rho(R)}=\mathrm{e}^{\psi(\alpha)}$,

which gives a more physical parametrization of the solutions. For $\alpha \rightarrow 0$, we can use the asymptotic behavior $\psi \sim \frac{1}{6} \alpha^{2}$ of the potential at the origin (Eq. (23)) and we find $\frac{\beta G M}{R} \sim \frac{\alpha^{2}}{3} \rightarrow 0,-\frac{E R}{G M^{2}} \sim-\frac{9}{2 \alpha^{2}} \rightarrow-\infty$ and $\mathcal{R} \rightarrow 1$. This corresponds to high temperatures $T \rightarrow+\infty$. In this case, self-gravity is negligible with respect to thermal motion and the system behaves like an ordinary gas with uniform density. We check, by eliminating $\alpha$ from the two 
foregoing relations, that $E=\frac{3 M}{2 \beta}$. This is indeed the equation of state for an ideal gas without self-gravity. When we proceed along the spiral, the density contrast increases monotonically and goes to $+\infty$ at the center of the spiral. Using the asymptotic expansion $\mathrm{e}^{-\psi(\alpha)} \sim \frac{2}{\alpha^{2}}$ of the potential at large distances (Eq. (24)), we find $\frac{\beta G M}{R} \rightarrow 2$, $-\frac{E R}{G M^{2}} \rightarrow \frac{1}{4}$ and $\mathcal{R} \sim \frac{\alpha^{2}}{2} \rightarrow+\infty$. We can check by a direct calculation that the terminal point $(1 / 4,2)$ of the spiral corresponds to the singular sphere (18). Clearly, the spiral behavior of the $(\Lambda, \eta)$ curve can be ascribed to the oscillating behavior of the solution (24) as $\xi \rightarrow+\infty$. For large values of $\alpha$, the equation of the spiral is explicitly given by

$$
\begin{aligned}
\eta=2+\frac{A}{2 \alpha^{1 / 2}} & {\left[\sqrt{7} \sin \left(\frac{\sqrt{7}}{2} \ln \alpha+\delta\right)\right.} \\
+ & \left.\cos \left(\frac{\sqrt{7}}{2} \ln \alpha+\delta\right)\right],
\end{aligned}
$$

$$
\begin{array}{r}
\Lambda=\frac{1}{4}-\frac{A}{16 \alpha^{1 / 2}}\left[7 \cos \left(\frac{\sqrt{7}}{2} \ln \alpha+\delta\right)\right. \\
\left.-\sqrt{7} \sin \left(\frac{\sqrt{7}}{2} \ln \alpha+\delta\right)\right] .
\end{array}
$$

\subsection{The Milne variables}

It will be convenient in the following to introduce the Milne variables $(u, v)$ defined by (Chandrasekhar 1942):

$u=\frac{\xi \mathrm{e}^{-\psi}}{\psi^{\prime}} \quad$ and $\quad v=\xi \psi^{\prime}$.

Taking the logarithmic derivative of $u$ and $v$ with respect to $\xi$ and using Eq. (20), we get

$\frac{1}{u} \frac{\mathrm{d} u}{\mathrm{~d} \xi}=\frac{1}{\xi}(3-v-u)$

$\frac{1}{v} \frac{\mathrm{d} v}{\mathrm{~d} \xi}=\frac{1}{\xi}(u-1)$.

Taking the ratio of the foregoing equations, we find that the variables $u$ and $v$ are related to each other by a first order differential equation

$\frac{u}{v} \frac{\mathrm{d} v}{\mathrm{~d} u}=-\frac{u-1}{u+v-3}$.

Therefore, by using the Milne variables, the degree of the meanfield Eq. (20) has been reduced from two to one. As discussed extensively by Chandrasekhar (1942), this property is related to the homology invariance of the solutions of the Emden equation. The solution curve in the $(u, v)$ plane is well known and is ploted in Fig. 4. The curve is parametrized by $\xi$. Starting from the point $(u, v)=(3,0)$ with a slope $(\mathrm{d} v / \mathrm{d} u)_{0}=-5 / 3$, corresponding to $\xi=0$, the solution curve spirals indefinitely around the point $\left(u_{\mathrm{s}}, v_{\mathrm{s}}\right)=(1,2)$, corresponding to the singular sphere, as $\xi$ tends to infinity. All isothermal spheres must necessarily

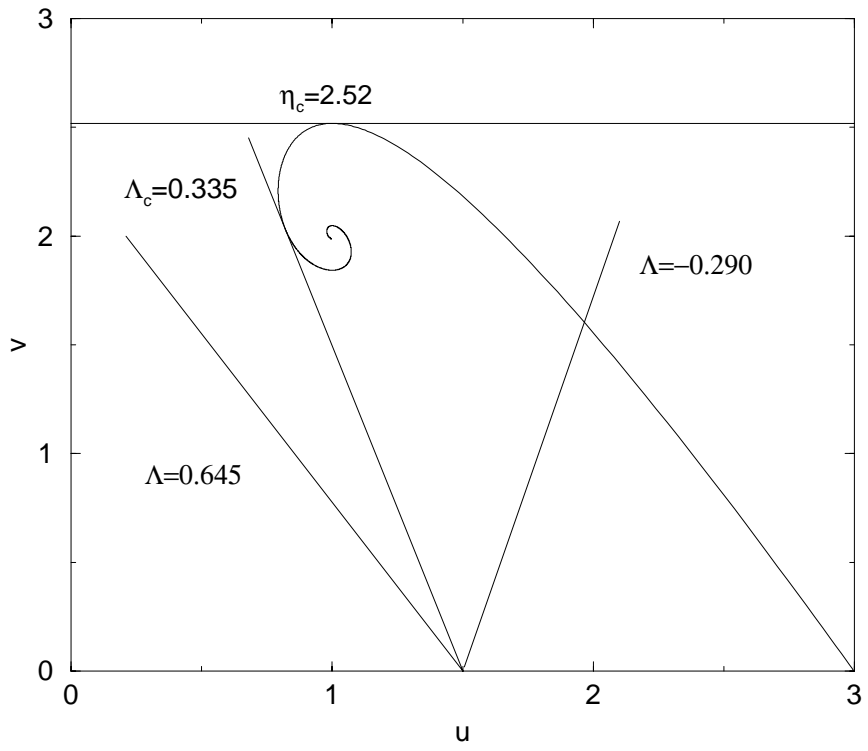

Fig. 4. The $(u, v)$ plane. All isothermal spheres must necessarily lie on the spiral. There exists solutions only for $\eta<2.52$ and $\Lambda<0.335$.

lie on this curve. For bounded isothermal spheres, $\xi$ must be terminated at the box radius $\alpha$.

It turns out that the normalized temperature and energy can be expressed very simply in terms of the values of $u$ and $v$ at the normalized box radius $\alpha$. Indeed, writing $u_{0}=u(\alpha)$ and $v_{0}=v(\alpha)$ and using Eqs. (25), (26), we get

$\Lambda=\frac{1}{v_{0}}\left(\frac{3}{2}-u_{0}\right)$

$\eta=v_{0}$

The relation (34) was previously noticed by Padmanabhan (1989). The intersections between the straight lines defined by Eqs. (34), (35) and the spiral in the $(u, v)$ plane determine the values of $\alpha$ corresponding to a given energy or temperature. As noticed by Padmanabhan, for $\Lambda>\Lambda_{\mathrm{c}}=0.335$ there is no intersection. Thus, in the microcanonical ensemble, no isothermal sphere can exist if $-\frac{E R}{G M^{2}}>0.335$. This result can also be read from Fig. 2 and was discovered by Antonov (1962). For sufficiently small energies, the system is expected to collapse and overheat in agreement with the "gravothermal catastrophe" picture (Lynden-Bell \& Wood 1968). Similarly, considering Eq. (35), we find that there is no intersection for $\frac{\beta G M}{R}>v_{\max }=2.52$ (where $v_{\max }$ corresponds to $u=1$ ). This result can also be read from Fig. 3. In the canonical ensemble, a gaseous sphere is expected to collapse below a critical temperature $k T_{\mathrm{c}}=\frac{G m M}{2.52 R}$ (Lynden-Bell \& Wood 1968).

\subsection{The stability analysis of Katz}

More precisely, it is possible to prove the following stability result: when a self-gravitating system is confined within a box and maintained at a constant temperature 
$T$, local maxima of free energy exist only for $\frac{\beta G M}{R} \leq 2.52$; they have a density contrast $\mathcal{R}=\rho(0) / \rho(R)<32.1$ (and $\alpha<8.99$ ). Critical points of free energy with density contrast $\mathcal{R}>32.1$ (and $\alpha>8.99$ ) are unstable saddle points. For $\frac{\beta G M}{R}>2.52$, there are no critical points of free energy.

The stability of the solutions can be deduced from the topology of the $E-\beta$ curve by using the method of Katz (1978) who has extended Poincaré's theory of linear series of equilibrium. The parameter conjugate to the free energy with respect to the inverse temperature $\beta$ is $-E=\left(\frac{\partial J}{\partial \beta}\right)_{M, R}$. Then, if we plot $E$ as a function of $\beta$ (we just need to rotate Fig. 3 by $90^{\circ}$ ) we have the following results: (i) a change of stability can occur only at a limit point where $\beta$ is an extremum $(\mathrm{d} E / \mathrm{d} \beta$ infinite) (ii) a mode of stability is lost when the curve rotates clockwise and gained otherwise. Now, we know that for $T$ sufficiently large the solutions are stable because, in this limit, self-gravity is negligible and the system behaves like an ordinary gas. From point (i) we conclude that the solutions with $\mathcal{R}=\rho(0) / \rho(R)<32.1$ are stable. As the curve spirals inward for $\mathcal{R}>32.1$, more and more modes of stability are lost. In this respect, the singular sphere, at the end of the spiral, is the most unstable solution ${ }^{1}$. It can be noted that instability sets in precisely when the specific heat $C=\mathrm{d} E / \mathrm{d} T$ becomes negative (in the range $32.1<\mathcal{R}<709)$. General considerations indicate that negative specific heats are forbidden in the canonical ensemble (Padmanabhan 1990). By contrast, thermally isolated systems can have negative specific heats: in the microcanonical ensemble, the solutions with $32.1<\mathcal{R}<709$ are stable (Katz 1978; Padmanabhan 1989). This clearly demonstrates that the statistical ensembles do not coincide for self-gravitating systems: the region of negative specific heats in the microcanonical ensemble is replaced by a phase transition (an "isothermal collapse") in the canonical ensemble (Padmanabhan 1990). It is at the verge of this phase transition that the meanfield approximation ceases to be valid as demonstrated by de Vega et al. (2001a, 2001b).

\subsection{Analogy with critical phenomena}

In this section, we study some analogies with the theory of critical phenomena. Further analogies are discussed in Chavanis et al. (2001) where a simple dynamical model for studying phase transitions in self-gravitating systems is proposed.

The specific heat can be written

$C=\frac{\mathrm{d} E}{\mathrm{~d} T}=-\frac{k}{m} \beta^{2} \frac{\mathrm{d} E}{\mathrm{~d} \beta}=N k \eta^{2} \frac{\mathrm{d} \Lambda}{\mathrm{d} \eta}$.

Using Eqs. (34), (35), (31), and (32), we easily get

$\frac{\mathrm{d} \eta}{\mathrm{d} \alpha}=\frac{v_{0}}{\alpha}\left(u_{0}-1\right)$

1 Another application of Katz theorem where the spiral unwinds and the stability is regained is given by Chavanis \& Sommeria (1998) for self-gravitating fermions.

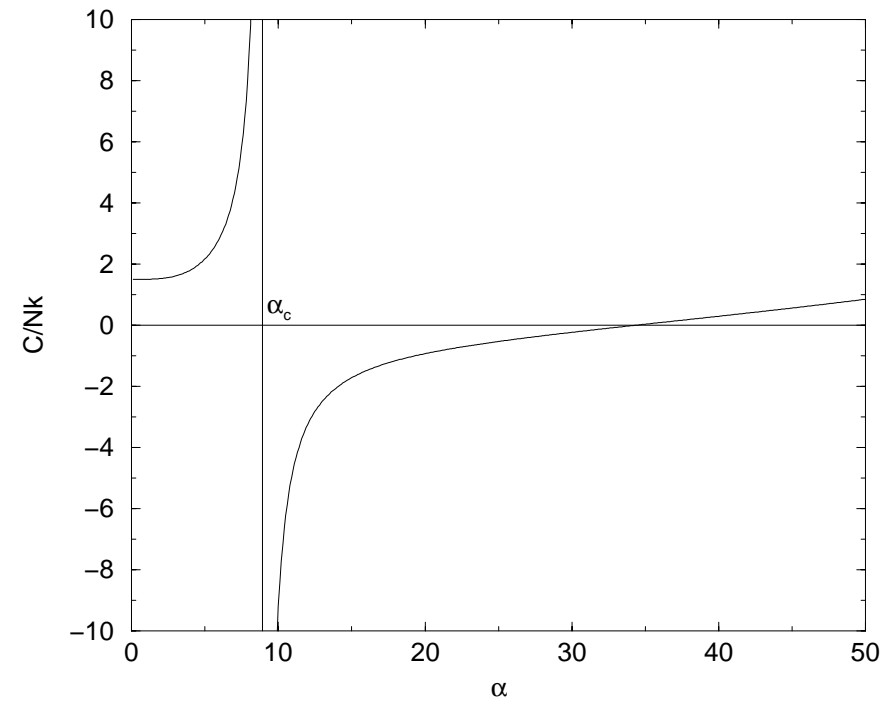

Fig. 5. Evolution of the specific heat as a function of $\alpha$. The specific heat diverges for the first time at $\alpha_{c}=8.99$ and is negative in the range $8.99<\alpha<34.4$. For $\alpha=0, C=\frac{3}{2} N k$ (perfect gas).

$\frac{\mathrm{d} \Lambda}{\mathrm{d} \alpha}=\frac{1}{2 \alpha v_{0}}\left(4 u_{0}^{2}+2 u_{0} v_{0}-11 u_{0}+3\right)$.

Therefore, the specific heat is determined as a function of $\alpha$ by the expression

$C=N k \frac{4 u_{0}^{2}+2 u_{0} v_{0}-11 u_{0}+3}{2\left(u_{0}-1\right)}$.

Of course, $C \rightarrow \infty$ if $\mathrm{d} \eta=0$ and $C \rightarrow 0$ if $\mathrm{d} \Lambda=0$. On the other hand, for the ideal gas $\left(u_{0}, v_{0}\right)=(3,0)$, we recover the well-known result $C=\frac{3}{2} N k$. The specific heat is ploted as a function of $\alpha$ in Fig. 5 .

At the critical point $\eta=\eta_{\mathrm{c}}$ at which $C$ diverges, we write $\alpha=\alpha_{\mathrm{c}}+\epsilon$ and expand $\eta$ and $\Lambda$ in terms of $\epsilon$, using Eqs. (37), (38) and $u_{0}\left(\alpha_{\mathrm{c}}\right)=1, v_{0}\left(\alpha_{\mathrm{c}}\right)=\eta_{\mathrm{c}}$. To lowest order, we have

$\eta-\eta_{\mathrm{c}}=\left(\frac{\mathrm{d}^{2} \eta}{\mathrm{d} \alpha^{2}}\right)_{\alpha_{\mathrm{c}}} \frac{\epsilon^{2}}{2}=\frac{\eta_{\mathrm{c}}}{2 \alpha_{\mathrm{c}}^{2}}\left(2-\eta_{\mathrm{c}}\right) \epsilon^{2}$,

$\Lambda-\Lambda_{0}=\left(\frac{\mathrm{d} \Lambda}{\mathrm{d} \alpha}\right)_{\alpha_{\mathrm{c}}} \epsilon=\frac{\eta_{\mathrm{c}}-2}{\alpha_{\mathrm{c}} \eta_{\mathrm{c}}} \epsilon$

where $\Lambda_{0} \simeq 0.197$ is the value of $\Lambda$ at $\alpha=\alpha_{\mathrm{c}}$. Eliminating $\epsilon$ from these expressions, we find the relation between $\eta$ and $\Lambda$ close to the critical point

$\eta_{\mathrm{c}}-\eta=\frac{\eta_{\mathrm{c}}^{3}}{2\left(\eta_{\mathrm{c}}-2\right)}\left(\Lambda-\Lambda_{0}\right)^{2}$

Then, using Eq. (36), we find explicitly

$C=\left\{\begin{array}{c}-N k \frac{\eta_{\mathrm{c}}-2}{\eta_{\mathrm{c}}}\left(\Lambda-\Lambda_{0}\right)^{-1}, \\ \pm N k\left[\frac{\eta_{\mathrm{c}}\left(\eta_{\mathrm{c}}-2\right)}{2}\right]^{1 / 2}\left(\eta_{\mathrm{c}}-\eta\right)^{-1 / 2} .\end{array}\right.$

The specific heat diverges as a function of the energy with an exponent $\nu=1$ and as a function of the temperature 


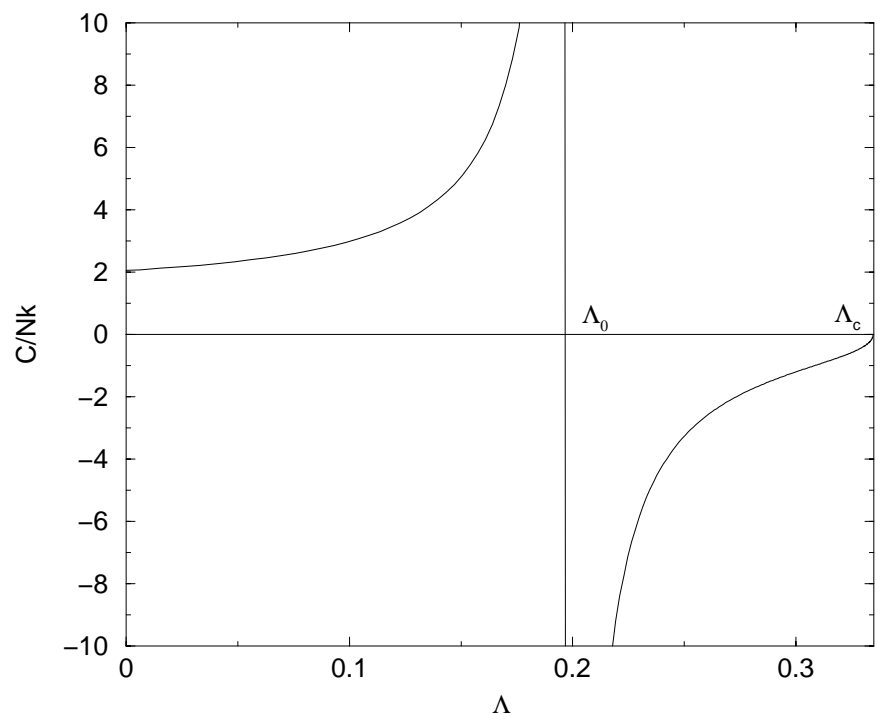

Fig. 6. Evolution of the specific heat as a function of $\Lambda$ near the point $\Lambda_{0}$. The specific heat diverges like $C \sim-\left(\Lambda-\Lambda_{0}\right)^{-1}$. It is negative for $\Lambda>\Lambda_{0}$ and tends to $\frac{3}{2} N k$ for $\Lambda \rightarrow-\infty$ (high energies). The qualitative behaviour of this curve is consistent with the microcanonical numerical simulations of Cerruti-Sola et al. (2001) who solved the gravitational $N$-body problem in a finite container.

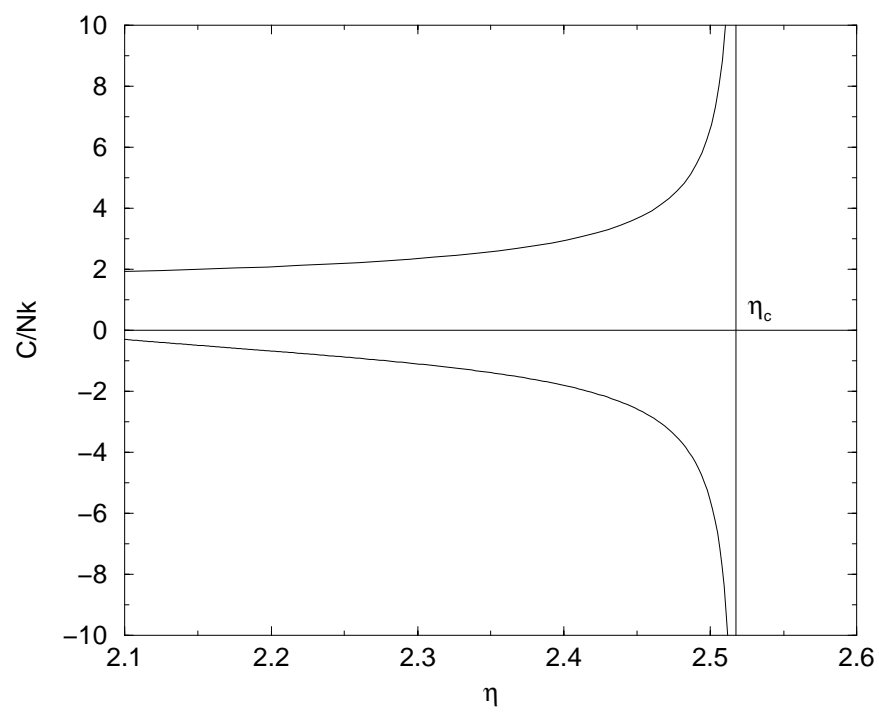

Fig. 7. Evolution of the specific heat as a function of $\eta$ near the critical point $\eta_{c}$. The specific heat diverges like $C \sim \pm\left(\eta_{c}-\right.$ $\eta)^{-1 / 2}$. It tends to $\frac{3}{2} N k$ for $\eta \rightarrow 0$ (high temperatures). The region of negative specific heat (lower curve) is forbidden in the canonical ensemble.

with an exponent $\nu^{\prime}=1 / 2$ (see Figs. 6, 7). It becomes negative for $\Lambda_{0}<\Lambda<\Lambda_{\mathrm{c}}$. This region of negative specific heats is allowed in the microcanonical ensemble but not in the canonical ensemble.

It is also of interest to determine the behavior of the central density $\rho_{0}$ near the critical point (C. Sire, private communication). The central density is related to $\alpha$ by the relation $\alpha=\left(4 \pi G \beta \rho_{0}\right)^{1 / 2} R$ or, alternatively,

$$
\rho_{0}=\frac{\alpha^{2} M}{4 \pi R^{3} \eta} .
$$

To first order in $\epsilon$, we have

$\rho_{0}-\rho_{0}^{\mathrm{c}}=\frac{2 \alpha_{\mathrm{c}} M}{4 \pi R^{3} \eta_{\mathrm{c}}} \epsilon$.

where $\rho_{0}^{\mathrm{c}}=\alpha_{\mathrm{c}}^{2} M /\left(4 \pi R^{3} \eta_{\mathrm{c}}\right)$ is the value of the central density at the critical point $\eta_{\mathrm{c}}$. Using Eqs. (40), (41), we get

$$
\frac{\rho_{0}^{\mathrm{c}}-\rho_{0}}{\rho_{0}^{\mathrm{c}}}=\left\{\begin{array}{c}
-\frac{2 \eta_{\mathrm{c}}}{\eta_{\mathrm{c}}-2}\left(\Lambda-\Lambda_{0}\right), \\
\pm\left[\frac{8}{\eta_{\mathrm{c}}-2}\left(1-\frac{\eta}{\eta_{\mathrm{c}}}\right)\right]^{1 / 2} .
\end{array}\right.
$$

As shown by Chavanis et al. (2001), this stationary result can also be derived from dynamical considerations. Note finally that, using Eqs. (27), (30), we find $\mathcal{R}_{\mathrm{c}}=\alpha_{\mathrm{c}}^{2} / \eta_{\mathrm{c}}$, which implies $\rho^{\mathrm{c}}(R)=M /\left(4 \pi R^{3}\right)=\bar{\rho} / 3$.

\section{An extension of Padmanabhan's stability analysis}

\subsection{The condition of thermodymamical stability}

The stability of the gaseous spheres can be studied by extending Padmanabhan (1989)'s stability analysis to the canonical ensemble. This will provide the profiles of the perturbations that induce instability.

A critical point of free energy is a local maximum if, and only if, the second variations

$\delta^{2} J=-\frac{k}{m} \int \frac{(\delta \rho)^{2}}{2 \rho} \mathrm{d}^{3} \boldsymbol{r}-\frac{1}{2 T} \int \delta \rho \delta \Phi \mathrm{d}^{3} \boldsymbol{r}$,

are negative for any variation $\delta \rho$ that conserves mass to first order. This conservation law imposes

$\int \delta \rho \mathrm{d}^{3} \boldsymbol{r}=0$

We shall restrict ourselves to spherically symmetric perturbations since only spherically symmetric perturbations can induce instability for non rotating systems. Following Padmanabhan (1989), we introduce the variable $q$ defined by

$\delta \rho=\frac{1}{4 \pi r^{2}} \frac{\mathrm{d} q}{\mathrm{~d} r}$

Physically, $q$ represents the mass perturbation $q(r) \equiv$ $\delta M(r)=\int_{0}^{r} 4 \pi r^{\prime 2} \delta \rho\left(r^{\prime}\right) \mathrm{d} r^{\prime}$ within the sphere of radius $r$. The boundary conditions on $q$ are thus

$q(0)=q(R)=0$.

Substituting the foregoing expression for $q(r)$ in Eq. (47), we obtain

$\delta^{2} J=-\frac{1}{2 T} \int_{0}^{R} \frac{\mathrm{d} q}{\mathrm{~d} r} \delta \Phi \mathrm{d} r-\frac{k}{m} \int_{0}^{R} \frac{1}{8 \pi \rho r^{2}}\left(\frac{\mathrm{d} q}{\mathrm{~d} r}\right)^{2} \mathrm{~d} r$ 
Integrating by parts and using the boundary conditions (50), we get

$\delta^{2} J=\frac{1}{2 T} \int_{0}^{R} q \frac{\mathrm{d} \delta \Phi}{\mathrm{d} r} \mathrm{~d} r+\frac{k}{m} \int_{0}^{R} q \frac{\mathrm{d}}{\mathrm{d} r}\left(\frac{1}{8 \pi \rho r^{2}} \frac{\mathrm{d} q}{\mathrm{~d} r}\right) \mathrm{d} r$.

Then, using Gauss theorem in the form

$\frac{\mathrm{d} \delta \Phi}{\mathrm{d} r}=\frac{G q}{r^{2}}$

we find

$\delta^{2} J=\frac{1}{2 T} \int_{0}^{R} \frac{G q^{2}}{r^{2}} \mathrm{~d} r+\frac{k}{m} \int_{0}^{R} q \frac{\mathrm{d}}{\mathrm{d} r}\left(\frac{1}{8 \pi \rho r^{2}} \frac{\mathrm{d} q}{\mathrm{~d} r}\right) \mathrm{d} r$,

or, equivalently,

$\delta^{2} J=\frac{1}{2} \int_{0}^{R} \mathrm{~d} r q\left[\frac{G}{T r^{2}}+\frac{k}{m} \frac{\mathrm{d}}{\mathrm{d} r}\left(\frac{1}{4 \pi \rho r^{2}} \frac{\mathrm{d}}{\mathrm{d} r}\right)\right] q$.

The second variations of free energy can be positive (implying instability) only if the differential operator which occurs in the integral has positive eigenvalues. We need therefore to consider the eigenvalue problem

$\left[\frac{k}{m} \frac{\mathrm{d}}{\mathrm{d} r}\left(\frac{1}{4 \pi \rho r^{2}} \frac{\mathrm{d}}{\mathrm{d} r}\right)+\frac{G}{T r^{2}}\right] q_{\lambda}(r)=\lambda q_{\lambda}(r)$,

with $q_{\lambda}(0)=q_{\lambda}(R)=0$. If all the eigenvalues $\lambda$ are negative, then the critical point is a maximum of free energy. If at least one eigenvalue is positive, the critical point is an unstable saddle point. The point of marginal stability $\eta=\eta_{\mathrm{c}}$ is determined by the condition that the largest eigenvalue is equal to zero. We thus have to solve the differential equation

$\frac{k}{m} \frac{\mathrm{d}}{\mathrm{d} r}\left(\frac{1}{4 \pi \rho r^{2}} \frac{\mathrm{d} F}{\mathrm{~d} r}\right)+\frac{G F(r)}{T r^{2}}=0$,

with $F(0)=F(R)=0$. Note that this equation corresponds to that found by Padmanabhan (1989) in the microcanonical ensemble with $V=0$.

\subsection{The point of marginal stability}

Introducing the dimensionless variables of Sect. 2.4, we can rewrite the foregoing equation in the form

$\frac{\mathrm{d}}{\mathrm{d} \xi}\left(\frac{\mathrm{e}^{\psi}}{\xi^{2}} \frac{\mathrm{d} F}{\mathrm{~d} \xi}\right)+\frac{F(\xi)}{\xi^{2}}=0$,

with $F(0)=F(\alpha)=0$. As shown by Padmanabhan (1989), the function $F(\xi)$ can be expressed in terms of the solution $\psi(\xi)$ of the Emden Eq. (20). Let us introduce the differential operator

$\mathcal{L}=\frac{\mathrm{d}}{\mathrm{d} \xi}\left(\frac{\mathrm{e}^{\psi}}{\xi^{2}} \frac{\mathrm{d}}{\mathrm{d} \xi}\right)+\frac{1}{\xi^{2}}$.

Using Eq. (20), it is readily established that

$\mathcal{L}\left(\xi^{2} \psi^{\prime}\right)=\frac{\mathrm{d}}{\mathrm{d} \xi}\left(\frac{\mathrm{e}^{\psi}}{\xi^{2}} \frac{\mathrm{d}}{\mathrm{d} \xi}\left(\xi^{2} \psi^{\prime}\right)\right)+\psi^{\prime}=$
$\frac{\mathrm{d}}{\mathrm{d} \xi}\left(\mathrm{e}^{\psi} \times \mathrm{e}^{-\psi}\right)+\psi^{\prime}=\psi^{\prime}$
Similarly, we find

$\mathcal{L}\left(\xi^{3} \mathrm{e}^{-\psi}\right)=\frac{\mathrm{d}}{\mathrm{d} \xi}\left(\frac{\mathrm{e}^{\psi}}{\xi^{2}} \frac{\mathrm{d}}{\mathrm{d} \xi}\left(\xi^{3} \mathrm{e}^{-\psi}\right)\right)+\xi \mathrm{e}^{-\psi}$
$=\frac{\mathrm{d}}{\mathrm{d} \xi}\left(3-\xi \psi^{\prime}\right)+\xi \mathrm{e}^{-\psi}=-\xi \psi^{\prime \prime}-\psi^{\prime}+\xi \mathrm{e}^{-\psi}=\psi^{\prime}$.

These identities suggest to seek a solution of Eq. (58) in the form

$F(\xi)=c_{1} \xi^{3} \mathrm{e}^{-\psi}+c_{2} \xi^{2} \psi^{\prime}$

where $c_{1}$ and $c_{2}$ are constants. Substituting the foregoing expression for $F(\xi)$ in the differential Eq. (58), we find that $c_{1}+c_{2}=0$. The function $F(\xi)$ can therefore be reexpressed as

$F(\xi)=c_{1}\left(\xi^{3} \mathrm{e}^{-\psi}-\xi^{2} \psi^{\prime}\right)$.

The boundary condition $F(0)=0$ is automatically satisfied (we can show furthermore that Eq. (63) is the only solution consistent with this boundary condition). The condition $F(\alpha)=0$ will determine the point on the spiral of Fig. 3 at which the gaseous spheres become unstable (i.e., the point of marginal stability). From Eq. (63), we get

$\alpha^{3} \mathrm{e}^{-\psi(\alpha)}-\alpha^{2} \psi^{\prime}(\alpha)=0$.

This can be expressed in terms of the Milne variables (30) as

$u_{0}=1$.

Considering Eq. (33), we see that the condition (65) determines the points for which $v$ is extremum $(\mathrm{d} v / \mathrm{d} u=0)$. Since $\eta=v_{0}$ according to Eq. (35), we deduce that a change of stability occurs each time that the temperature is extremum (see Fig. 8) in complete agreement with the theorem of Katz (1978). In particular, the first instability (for which the largest eigenvalue $\lambda$ becomes positive) corresponds to $\eta_{\mathrm{c}}=v_{\max }=2.52$ (for this value $\alpha=8.99$ ). We show in the next section that the secondary instabilities occur at values of $\alpha$ that follow asymptotically a geometric progression. The profiles of the perturbations that induce these instabilities are discussed in Sect. 3.4.

\subsection{Secondary instabilities}

A mode of stability is lost each time that $u_{0}=u(\alpha)=1$. Now, it is easy to have an analytical estimate of $u(\xi)$ for large values of $\xi$. Introducing the asymptotic behavior (24) of $\psi$ in the Milne variable $u$ defined by Eq. (30) we get for $\xi \rightarrow+\infty$ :

$u=1+\frac{A}{4 \xi^{1 / 2}}\left[3 \cos \left(\frac{\sqrt{7}}{2} \ln \xi+\delta\right)-\sqrt{7} \sin \left(\frac{\sqrt{7}}{2} \ln \xi+\delta\right)\right]$.

Therefore, the condition $u_{0}=1$ corresponds to values of $\alpha$ satisfying

$\tan \left(\frac{\sqrt{7}}{2} \ln \alpha+\delta\right)=\frac{3}{\sqrt{7}} \quad(\alpha \rightarrow+\infty)$, 


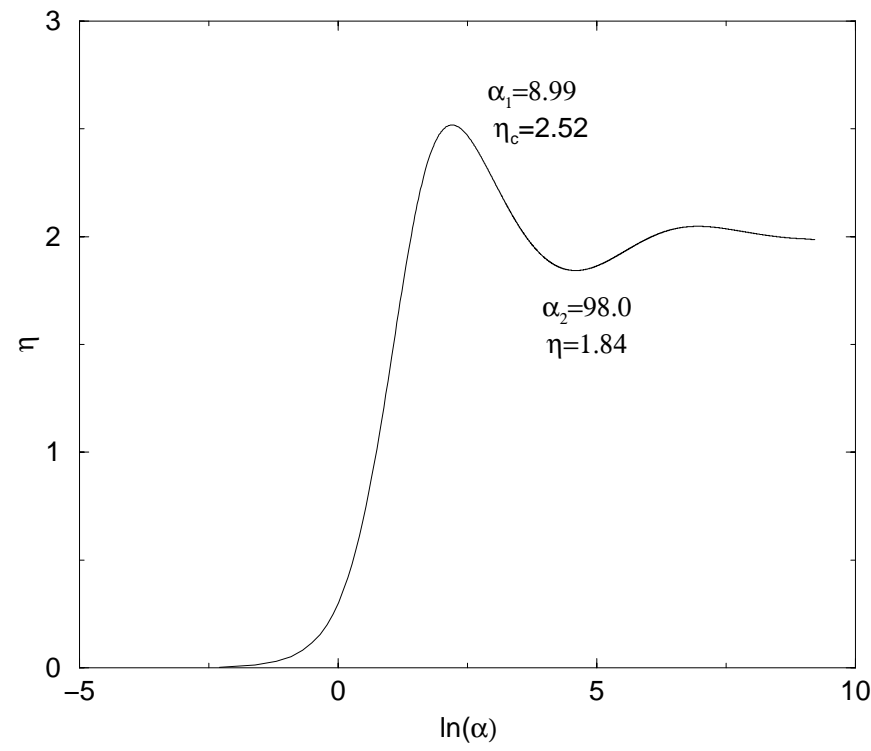

Fig. 8. Evolution of the inverse temperature $\eta$ as a function of the scaled radius $\alpha$. A mode of stability is lost each time that $\eta$ is extremum. This figure is the counterpart of Fig. 3 of Padmanabhan (1989) in the microcanonical ensemble.

or equivalently

$\frac{\sqrt{7}}{2} \ln \alpha_{n}+\delta=\arctan (3 / \sqrt{7})+n \pi \quad(n$ integer $)$.

This determines a succession of values that follow the geometric progression

$\alpha_{n} \sim \mathrm{e}^{\frac{2 \pi n}{\sqrt{7}}}=[10.74 \ldots]^{n} \quad(n \rightarrow+\infty$, integer $)$.

At these points a new eigenvalue becomes positive implying secondary instabilities. Clearly, this geometric progression can be traced back to the "curious" asymptotic behavior of the solutions governing the isothermal gas sphere (see Eq. (24)) which intersects the singular solution (21) infinitely often at points that asymptotically increase geometrically in the ratio $1: 10.74$.

A mode of stability is lost each time that $\alpha$ achieves one of the values given by Eq. (69). If one prefers, the same criterion can be expressed in terms of the density contrast $\mathcal{R}$. Using Eq. (27) and the asymptotic expansion (24) we get $\mathcal{R}=\mathrm{e}^{\psi(\alpha)} \sim \frac{\alpha^{2}}{2}$ for $\alpha \rightarrow+\infty$. Hence, the result (69) is translated in

$\mathcal{R}_{n} \sim \mathrm{e}^{\frac{4 \pi n}{\sqrt{7}}}=[115.5 \ldots]^{n} \quad(n \rightarrow+\infty$, integer $)$.

\subsection{Perturbation profiles at the critical points}

In this section, we study the form of the perturbations that trigger instability at the critical points. Following the method of Padmanabhan (1989), it is possible to describe the behavior of these perturbation profiles without numerical work. Indeed, according to Eq. (49), the eigenfunction associated with the eigenvalue $\lambda=0$ can be written

$\frac{\delta \rho}{\rho_{0}}=\frac{1}{4 \pi \xi^{2}} \frac{\mathrm{d} F}{\mathrm{~d} \xi}$

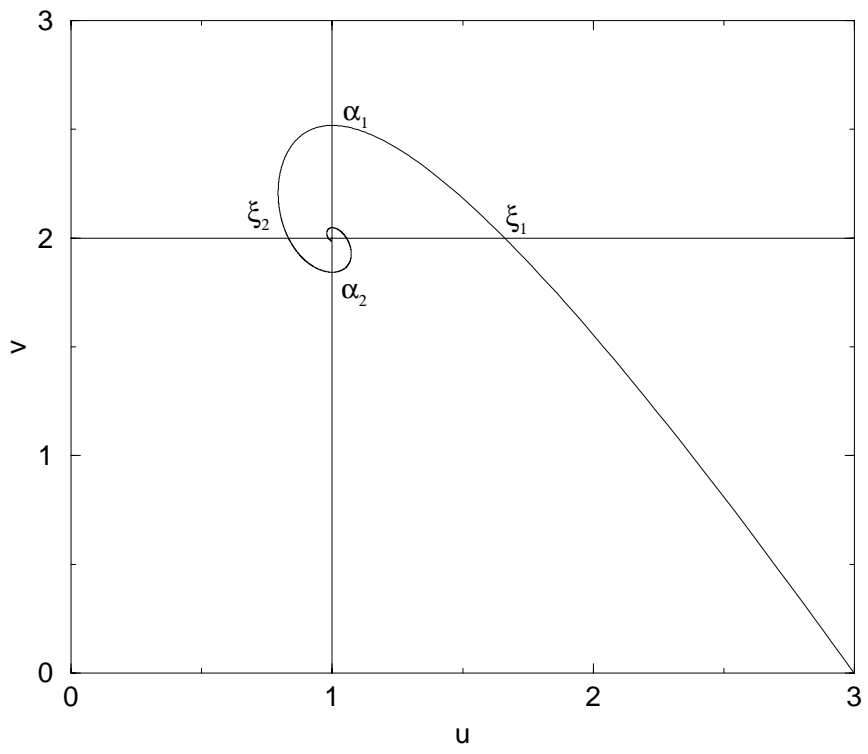

Fig. 9. Position of the zeros of the perturbation profile $\delta \rho / \rho$ in the $(u, v)$ plane.

where $F(\xi)$ is given by Eq. (63). Substituting this result in Eq. (71) and simplifying the derivative with the aid of Eq. (20) we obtain

$\frac{\delta \rho}{\rho}=\frac{c_{1}}{4 \pi}\left(2-\xi \psi^{\prime}\right)$.

Introducing the Milne variable $v$ defined by Eq. (30), we can rewrite the foregoing equation in the form

$\frac{\delta \rho}{\rho}=\frac{c_{1}}{4 \pi}(2-v)$.

The density perturbation $\delta \rho$ becomes zero at the point(s) $\xi_{i}$ such that $v\left(\xi_{i}\right)=2$. The number of zeros is therefore given by the number of intersections between the spiral in the $(u, v)$ plane and the line $v=2$ (see Fig. 9). For the first mode of instability $\left(\alpha_{1}=8.99\right)$, we need to follow the spiral until the first extremum of $v$, which corresponds to the point at which the box terminates (recall that $u\left(\alpha_{1}\right)=1$, see Sect. 3.2). In that case there is only one intersection $\xi_{1}$ with the line $v=2$. Therefore, the first mode of instability does not present a "core-halo" structure (Fig. 10), contrary to what happens in the microcanonical ensemble (Padmanabhan 1989). For the second mode of instability $\left(\alpha_{2}=98.0\right)$, we need to follow the spiral until the second extremum of $v$. The intersection with the line $v=2$ now determines two zeros $\xi_{1}$ and $\xi_{2}$, the first one being the same as for the first mode of instability. The profile of the second mode of instability therefore has a "core-halo" structure (Fig. 11). When we spiral inward, more and more intersections are obtained. Therefore the high order modes of instability present more and more oscillations. For these modes, it is easy to determine the asymptotic positions of their zeros. From Eqs. (30) and (24), we have for $\xi \rightarrow+\infty$ :

$v=2+\frac{A}{2 \xi^{1 / 2}}\left[\sqrt{7} \sin \left(\frac{\sqrt{7}}{2} \ln \xi+\delta\right)+\cos \left(\frac{\sqrt{7}}{2} \ln \xi+\delta\right)\right]$. 


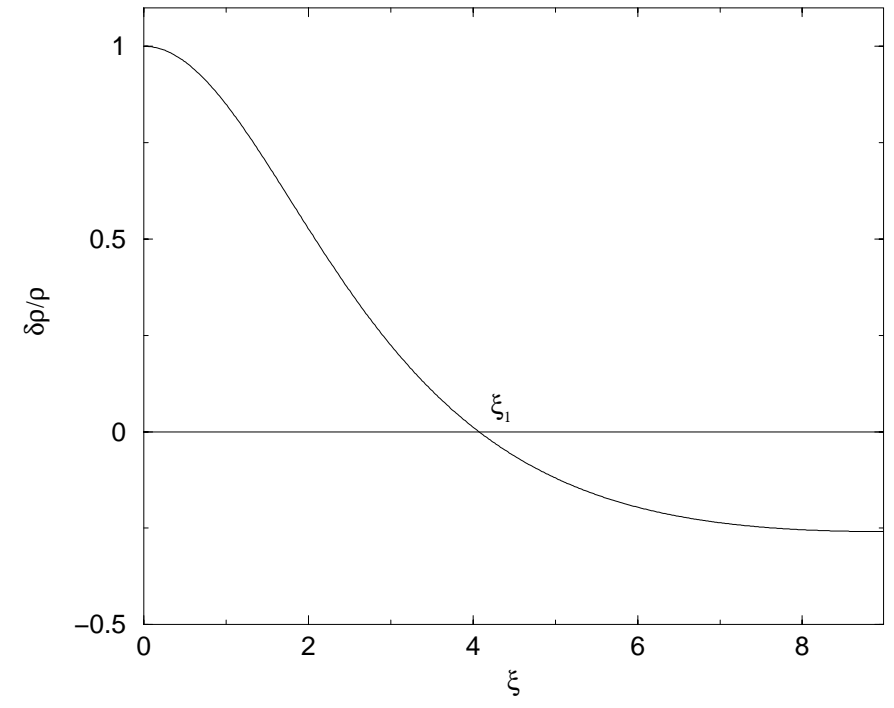

Fig. 10. First mode of instability corresponding to $\alpha_{1}=8.99$.

Thus, substituting $v=2$ in Eq. (74), we get

$\frac{\sqrt{7}}{2} \ln \xi_{i}+\delta=-\arctan (1 / \sqrt{7})+i \pi \quad(i$ integer $)$.

Therefore, the zeros follow asymptotically the geometric progression $^{2}$

$\xi_{i} \sim \mathrm{e}^{\frac{2 \pi i}{\sqrt{7}}}=[10.74 \ldots]^{i} \quad(i \rightarrow+\infty, i$ integer $)$.

Note that the perturbation that destabilizes the singular sphere (at the end of the spiral) has an infinite number of oscillations!

\section{Dynamical stability of isothermal gaseous spheres}

We shall now investigate the dynamical stability of isothermal spheres described by Navier-Stokes equations and compare the results with the thermodynamical approach. This problem was previously addressed by Yabushita (1968) in the isobaric ensemble and by Semelin et al. (2001) in the canonical ensemble. We shall prove analytically and with no approximation the equivalence between dynamical and thermodynamical stability and the fact that the stability of isothermal spheres does not depend on the viscosity. We shall also describe the behavior of the velocity profiles at the critical points.

The equations of the problem are the equation of continuity, the equation of motion and the Poisson equation

$\underline{\frac{\partial \rho}{\partial t}+\nabla(\rho \boldsymbol{v})}=0$

${ }^{2}$ It might be recalled, parenthetically, that the positions of the planets in the solar system also follow a geometric progression, but with a different ratio $q \simeq 2$ (Titius-Bode law). This is recognized to be an effect of scale invariance (Graner \& Dubrulle 1994; Dubrulle \& Graner 1994). There is, however, no real connexion with the present work and planets may have formed quite differently (see Chavanis 2000 and references therein).

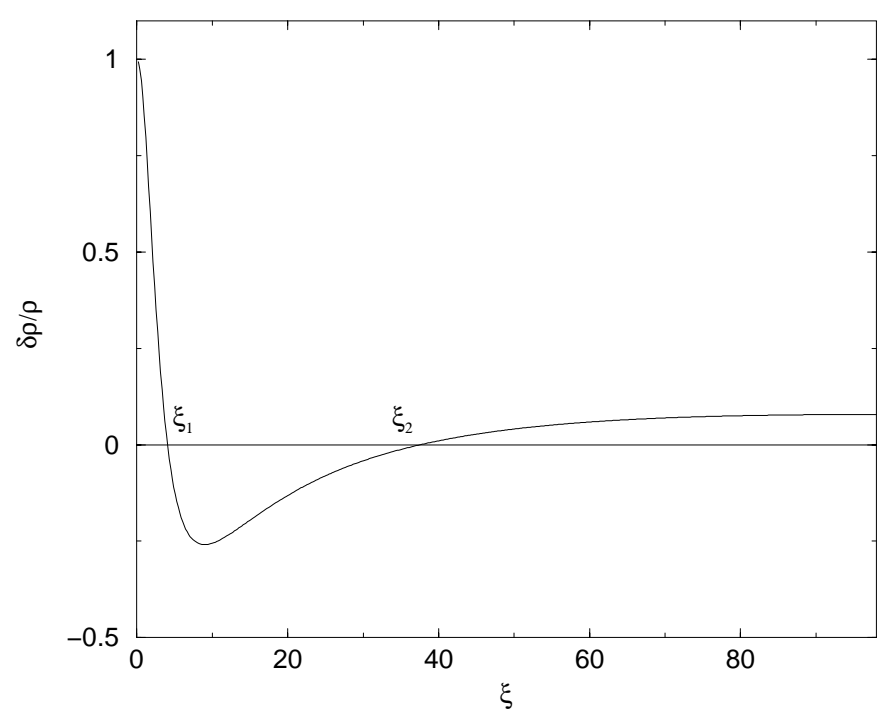

Fig. 11. Second mode of instability corresponding to $\alpha_{2}=$ 98.0 .

$\frac{\partial \boldsymbol{v}}{\partial t}+(\boldsymbol{v} \nabla) \boldsymbol{v}=-\frac{1}{\rho} \nabla p-\nabla \Phi+\frac{\eta}{\rho} \Delta \boldsymbol{v}+\frac{1}{\rho}\left(\zeta+\frac{\eta}{3}\right) \nabla(\nabla \boldsymbol{v})$,

$\Delta \Phi=4 \pi G \rho$.

They must be completed by an equation of state that we take of the form $p=\rho \frac{k}{m} T$ where $T$ is constant. We also recall that we work within a box of radius $R$. For astrophysical fluids, the Reynolds numbers are so huge that we can neglect the viscosity in the Navier-Stokes equation. We shall take therefore $\eta=\zeta=0$ in most of the calculations but we will also prove that viscosity does not change the onset of instability.

Clearly, the stationary solutions of Eqs. (77)-(79) correspond to the isothermal gaseous spheres studied in the previous sections. It should be recalled that the condition of mechanical equilibrium corresponds to the balance between the pressure force and the gravitational force. For an isothermal gas, this yields

$\frac{k T}{m} \frac{\mathrm{d} \rho}{\mathrm{d} r}+\rho \frac{\mathrm{d} \Phi}{\mathrm{d} r}=0$.

We now consider a small perturbation around a stationary solution and write

$\boldsymbol{v}=\delta \boldsymbol{v}(\boldsymbol{r}, t), \quad \rho=\bar{\rho}+\delta \rho(\boldsymbol{r}, t)$,

$p=\bar{p}+\delta p(\boldsymbol{r}, t), \quad \Phi=\bar{\Phi}+\delta \Phi(\boldsymbol{r}, t)$,

where the bar refers to the stationary solution (in the following we shall drop the bar). The linearized equations for the perturbations are

$$
\begin{aligned}
& \rho \frac{\partial \delta \boldsymbol{v}}{\partial t}=-\frac{k T}{m} \nabla \delta \rho-\delta \rho \nabla \Phi-\rho \nabla \delta \Phi \\
& \frac{\partial \delta \rho}{\partial t}+\nabla(\rho \delta \boldsymbol{v})=0 \\
& \Delta \delta \Phi=4 \pi G \delta \rho .
\end{aligned}
$$


Since we work with exact stationary solutions of the fluid equations, that are inhomogeneous, we do not have to advocate the Jeans swindle (Binney \& Tremaine 1987). In this sense, our approach is more rigorous than the classical treatment of Jeans starting from an infinite and homogeneous medium ${ }^{3}$.

In the following, we restrict ourselves to radial perturbations. It is known that non-radial perturbations do not lead to new instabilities. Writing the time dependance of the perturbation in the form $\delta v \sim \mathrm{e}^{\lambda t}, \delta \rho \sim \mathrm{e}^{\lambda t}, \ldots$, the equations of the problem become

$\lambda \rho \delta v=-\frac{k T}{m} \frac{\mathrm{d} \delta \rho}{\mathrm{d} r}-\delta \rho \frac{\mathrm{d} \Phi}{\mathrm{d} r}-\rho \frac{\mathrm{d} \delta \Phi}{\mathrm{d} r}$,

$\lambda \delta \rho+\frac{1}{r^{2}} \frac{\mathrm{d}}{\mathrm{d} r}\left(\rho r^{2} \delta v\right)=0$,

$\frac{1}{r^{2}} \frac{\mathrm{d}}{\mathrm{d} r}\left(r^{2} \frac{\mathrm{d} \delta \Phi}{\mathrm{d} r}\right)=4 \pi G \delta \rho$.

Introducing the notation (49) and using the Gauss theorem (53) we see that the Poisson Eq. (88) is automatically satisfied. The continuity Eq. (87) becomes

$\frac{\lambda}{4 \pi r^{2}} \frac{\mathrm{d} q}{\mathrm{~d} r}+\frac{1}{r^{2}} \frac{\mathrm{d}}{\mathrm{d} r}\left(\rho r^{2} \delta v\right)=0$.

This equation is readily integrated. Using the boundary condition $q(0)=0$, we get

$\delta v=-\frac{\lambda}{4 \pi \rho r^{2}} q$.

Substituting this result back into Eq. (86), we obtain

$\frac{\lambda^{2}}{4 \pi r^{2}} q=\frac{k T}{m} \frac{\mathrm{d}}{\mathrm{d} r}\left(\frac{1}{4 \pi r^{2}} \frac{\mathrm{d} q}{\mathrm{~d} r}\right)+\frac{1}{4 \pi r^{2}} \frac{\mathrm{d} q}{\mathrm{~d} r} \frac{\mathrm{d} \Phi}{\mathrm{d} r}+\frac{G \rho}{r^{2}} q$.

Using the condition of mechanical equilibrium (80), the foregoing equation can be rewritten

$\frac{k}{m} \frac{\mathrm{d}}{\mathrm{d} r}\left(\frac{1}{4 \pi \rho r^{2}} \frac{\mathrm{d} q}{\mathrm{~d} r}\right)+\frac{G q}{T r^{2}}=\frac{\lambda^{2}}{4 \pi \rho T r^{2}} q$.

This is similar to the eigenvalue Eq. (56) associated with the second variations of the free energy. In particular they coincide for marginal stability $\lambda=0$. Therefore, dynamical and thermodynamical instability occur at the same point in the series of equilibrium. This was not obvious a priori since the Navier-Stokes equations without viscosity conserve the free energy. Taking into account a finite viscosity, we obtain instead of Eq. (92),

$$
\begin{array}{r}
\frac{k}{m} \frac{\mathrm{d}}{\mathrm{d} r}\left(\frac{1}{4 \pi \rho r^{2}} \frac{\mathrm{d} q}{\mathrm{~d} r}\right)+\frac{G q}{T r^{2}}=\frac{\lambda^{2}}{4 \pi \rho T r^{2}} q \\
-\frac{1}{\rho T}\left(\zeta+\frac{4 \eta}{3}\right) \frac{\mathrm{d}}{\mathrm{d} r}\left[\frac{1}{r^{2}} \frac{\mathrm{d}}{\mathrm{d} r}\left(\frac{\lambda q}{4 \pi \rho}\right)\right] .
\end{array}
$$

${ }^{3}$ It should be recalled, however, that the Jeans procedure is justified in a cosmological context if we take into account the expansion of the universe.
For $\lambda=0$ the viscous term cancels out. Therefore, viscosity does not change the onset of instability, neither does it alter the profile of the perturbation that triggers the instability.

The profiles of the density perturbation have already been discussed in Sect. 3.4. The profiles of the velocity perturbation at the critical points are given by Eq. (90). In order to work with dimensionless variables, we introduce the speed of sound

$c_{\mathrm{s}}^{2}=\frac{\mathrm{d} p}{\mathrm{~d} \rho}=\frac{k}{m} T=\frac{1}{\beta}$.

Note that $c_{\mathrm{s}}$ can be written $c_{\mathrm{s}}=\frac{1}{3} L_{\mathrm{K}} / t_{\text {dyn }}$ where $t_{\mathrm{dyn}}=$ $\left(4 \pi G \rho_{0}\right)^{-1 / 2}$ is a time scale comparable with the dynamical time and $L_{\mathrm{K}}$ is the King's length (Sect. 5). Then,

$\frac{\delta v}{c_{\mathrm{S}}}=-\frac{\lambda^{\prime}}{4 \pi \xi^{2}} \mathrm{e}^{\psi} F(\xi)$

where we have set $\lambda^{\prime}=\lambda t_{\text {dyn. }}$ Using Eqs. (63)-(30), we find

$\frac{\delta v}{c_{\mathrm{S}}}=-\frac{\lambda^{\prime}}{4 \pi} c_{1} \psi^{\prime} \mathrm{e}^{\psi}(u-1)$

We assume that we are just at the onset of the instability $\left(\lambda^{\prime}=0^{+}\right)$so that Eq. (96) is applicable with $\lambda^{\prime}>0$ (the velocity profile at marginal stability, i.e. $\lambda^{\prime}=0$, is simply $\delta v=0)$. Since $\psi^{\prime}(0)=0$, the velocity always vanishes at the center of the sphere $\delta v(\xi=0)=0$. The other zero(s) are determined by the condition $u\left(\xi_{i}\right)=1$. Since the critical points of instability are also characterized by $u(\alpha)=1$, see Sect. 3.2, we deduce that the velocity perturbation always vanishes at the wall: $\delta v(\xi=\alpha)=0$. This result is to be expected since the mass does not leave the sphere. The other zeros are determined by the intersections between the spiral in the $(u, v)$ plane and the line $u=1$ (see Fig. 9). They correspond precisely to the values of $\alpha$ at which a new mode of instability occurs, i.e. $\xi_{i}=\alpha_{i}$. It is therefore straightforward to determine the number of zeros in the velocity profile. For the first instability there are only two zeros at $\xi=0$ and $\xi=\alpha_{1}$. For the second instability there are three zeros at $\xi=0$, $\xi=\alpha_{1}$ and $\xi=\alpha_{2}$. For the $n$th instability there are $n+1$ zeros $\xi=0, \xi=\alpha_{1}, \xi=\alpha_{2}, \ldots, \xi=\alpha_{n}$. The exact velocity profiles given by Eq. (96) are displayed in Figs. 12, 13 for $n=1,2$. They are in agreement with the numerical results of Semelin et al. (2001). For high order modes of instability, the zeros follow asymptotically the geometric progression (69). Returning to dimensional variables, the zeros of the $n$th mode of instability are located at $r=0$ and $r_{i}=\left(\alpha_{i} / \alpha_{n}\right) R(i=1, \ldots, n)$.

\section{Fragmentation and fractal structure of an isothermal self-gravitating gas}

We shall now express the previous results in a more physical form and make some speculations about the fragmentation and the fractal structure of an isothermal selfgravitating gas. 


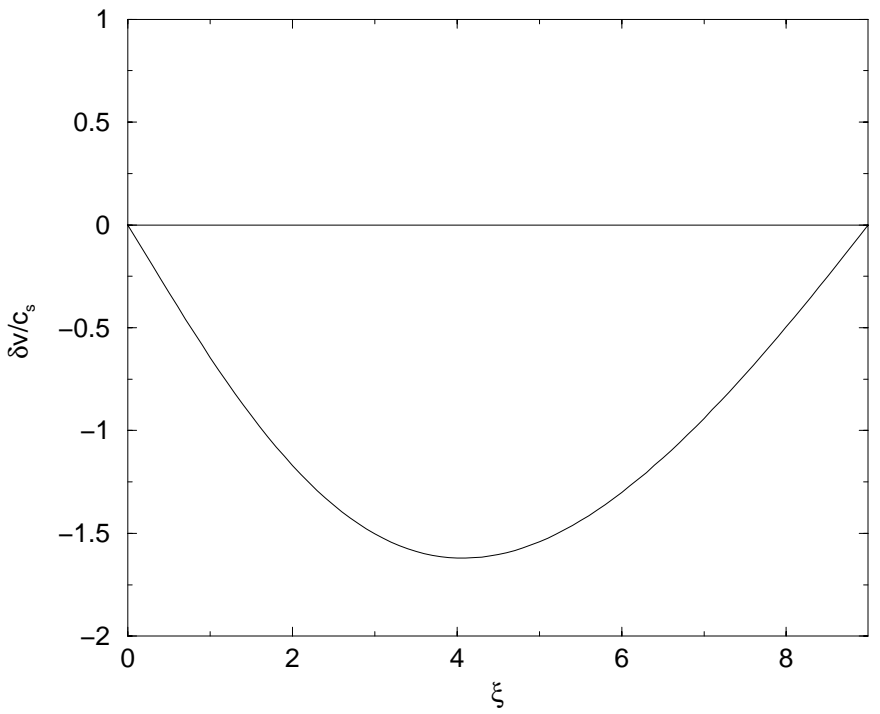

Fig. 12. Velocity profile for the first mode of instability corresponding to $\alpha_{1}=8.99$.

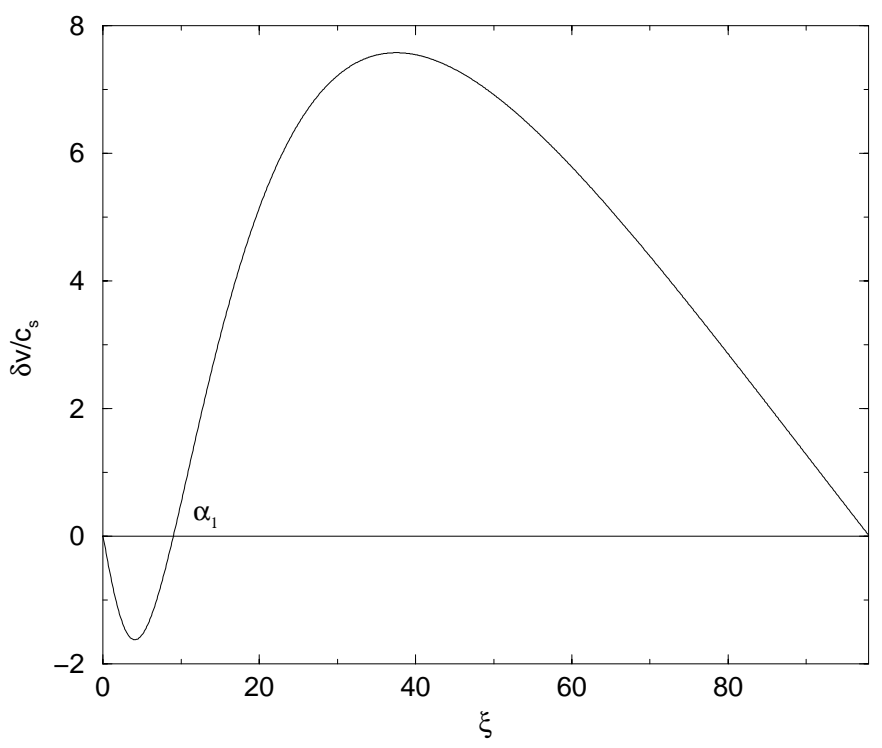

Fig. 13. Velocity profile for the second mode of instability corresponding to $\alpha_{2}=98.0$. We clearly see the separation between a core and a halo.

\subsection{The King's length}

Introducing the King's radius (Binney \& Tremaine 1987)

$$
L_{\mathrm{K}}=\left(\frac{9}{4 \pi G \beta \rho_{0}}\right)^{1 / 2} \text {, }
$$

the parameter $\alpha$ defined in Sect. 2.4 can be rewritten

$\alpha=3 \frac{R}{L_{\mathrm{K}}}$.

The King's radius gives a good estimate of the core radius of an isothermal sphere. In the following, we shall consider that the core radius $L_{\mathrm{K}}$ is fixed and that the domain size $R$ is increased. Clearly, this amounts to increasing the parameter $\alpha$ along the spiral. From the study of Sect. 3.2, we find that the onset of instability corresponds to

$R>\frac{8.99}{3} L_{\mathrm{K}}$

For larger radii there are still critical points of free energy but they are more and more unstable. According to Eq. (69), new modes of instability occur at radii that follow asymptotically the geometric progression

$R_{n} \sim[10.74 \ldots]^{n} L_{\mathrm{K}}$

This result is clearly related to that of Semelin et al. (1999) who first noticed the appearance of a hierarchy of scales connected to the instability of isothermal gaseous spheres. However, the connection with their work is not straightforward. Semelin et al. (1999) approximate the regular solutions of Eq. (17) by the analytical profile (18) and introduce an arbitrary short cut-off distance $\delta$ to avoid the central singularity. By contrast, there is no cut-off at small scales in our theory since we consider regular spheres that are exact solutions of the Emden equation (although we do not have analytical expressions for them). In this sense, there are no approximation nor indetermination in our theory. In fact, an "effective" cut-off is played by the King's length (or core radius) but it is not arbitrary and depends on physical parameters, the central density $\rho_{0}$ and the temperature $T$. For $r \gg L_{\mathrm{K}}$, the density profile behaves like $r^{-2}$ so that regular gaseous spheres can indeed be approximated by a $r^{-2}$ profile plus a cut-off $\delta$ at small scales as done by Semelin et al. (1999). Our approach suggests to take $\delta$ as the King's length. We have therefore given a new derivation of their result which is considerably simpler and does not introduce sophisticated renormalization group technics. It also avoids the introduction of an arbitrary cut-off. This provides, hopefully, an easier interpretation of their intriguing results.

According to Sect. 3.4, if we increase the domain size along the series of equilibrium (i.e. maintaining a fixed core radius) smaller and smaller regions become unstable. The modes that trigger these instabilities present numerous oscillations whose nodes also follow a geometric progression. These oscillations can be regarded as sort of "germs" leading to the formation of "clumps" preceding the fragmentation of the isothermal gas. It is expected that these "clumps" will evolve by achieving higher and higher density contrasts, and finally fragment in turn into substructures. This yields a hierarchy of structures fitting one into each other in a self-similar way. This picture is given further support by the fact that both the domain sizes that induce instability and the zeros of the perturbation profile in a given domain follow a geometric progression with the same ratio (see Eqs. (100), (76)). This property may explain in a quite natural fashion the fractal structure that a self-gravitating medium (e.g., the interstellar medium, the large scale structures of the universe, ...) can build under certain conditions. The hierarchy of scales that appears in an isothermal gas is due intrinsically to the spiral nature of the equilibrium phase diagram (see Figs. 2-4). 
This curious behavior has been known for a long time in the context of stellar structure (Chandrasekhar 1942) but it had not been apparently related to the geometric progression of scales inducing instability.

\subsection{The Jeans length}

We can also introduce another length scale defined with the average density $\bar{\rho}=\frac{3 M}{4 \pi R^{3}}$ of the system instead of the central density. This is the Jeans length (Binney \& Tremaine 1987)

$L_{\mathrm{J}}=\left(\frac{9}{4 \pi G \beta \bar{\rho}}\right)^{1 / 2}$.

The reduced temperature $\eta$ can be expressed in terms of the Jeans length as (de Vega \& Sanchez 2001a)

$\eta=\frac{\beta G M}{R}=3\left(\frac{R}{L_{\mathrm{J}}}\right)^{2}$.

In the following, we shall consider that the Jeans length $L_{\mathrm{J}}$ is fixed and that the domain size $R$ is increased. Clearly, this amounts to increasing the parameter $\eta$ along the vertical axis of Fig. 3. Using the results of Sect. 2.6, we find that the gaseous sphere becomes unstable when

$R>\sqrt{\frac{2.52 \ldots}{3}} L_{\mathrm{J}}$

For larger radii the system will collapse because there is no critical point of free energy (i.e. hydrostatic equilibrium). In that case, there are clearly no secondary instabilities. Indeed, when the condition (103) is realized we leave the spiral and loose the geometric hierarchy of scales associated with its turning points. Since the mode of instability at the critical point has no oscillation (see Sect. 3.4), we expect that the system will collapse without fragmenting, probably like in the study of Penston (1969).

\subsection{Fragmentation or not fragmentation?}

The fragmentation of the isothermal self-gravitating gas and the fractal structure that it can generate were previously discussed by de Vega et al. (1996a, 1996b, 1998) and we here improve and complete their physical arguments. In particular, the analytical calculation of the modes of instablity (without approximation), the geometric progression of both the domain sizes and the "clumps" sizes, and the precise physical picture that emerges from these results are new. In addition, we give arguments explaining why the instability of a self-gravitating gas does not always lead to a fragmentation process. In our model, the selection of the regime is related to how the size of the domain compares with the Jeans length or the King's length. If $R>0.916 \ldots L_{\mathrm{J}}$, we expect a collapse without fragmentation but if $R>2.996 \ldots L_{\mathrm{K}}$ (with a sufficiently large ratio) the medium is expected to fragment into several "clumps" corresponding to the oscillations of the perturbation profile.

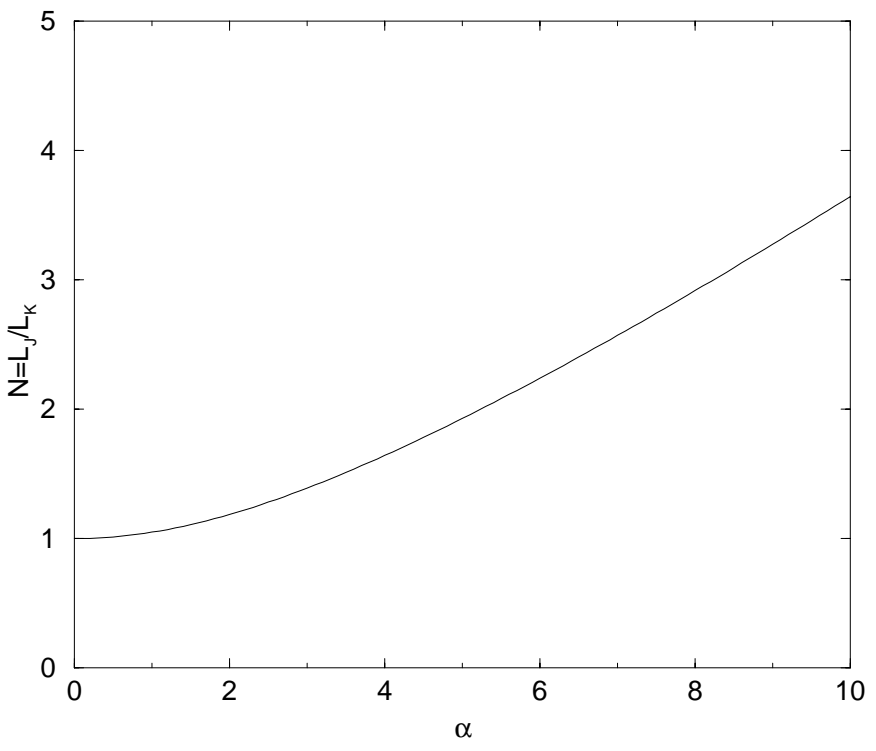

Fig. 14. Evolution of the dimensionless number $\mathcal{N}$ as a function of the scaled radius $\alpha$. This number quantifies the inhomogeneity of an isothermal self-gravitating gas.

The distinction between the Jeans length and the King's length is therefore essential in the present context and had not been noticed previously. In particular it is not the Jeans lengths but the King's lengths that follow a geometric progression (see formula (100)) contrary to the claim of Semelin et al. (1999). These two scales only coincide for a perfect gas with no self-gravity that is homogeneous. Indeed, for $\alpha \rightarrow 0$, we have $L_{\mathrm{J}} \sim L_{\mathrm{K}} \sim \frac{3 R}{\alpha} \rightarrow+\infty$. By contrast, for $\alpha \rightarrow+\infty$ (corresponding to the singular sphere) we get $L_{\mathrm{K}} \sim \frac{3 R}{\alpha} \rightarrow 0$ and $L_{\mathrm{J}} \rightarrow \sqrt{\frac{3}{2}} R$ (we have used the asymptotic expansions of Sect. 2.4). To appreciate the difference between the two length scales, it is convenient to introduce a dimensionless number

$\mathcal{N}=\frac{L_{\mathrm{J}}}{L_{\mathrm{K}}}$.

From the above formulae, we have the equivalent expressions

$\mathcal{N}=\left(\frac{\rho_{0}}{\bar{\rho}}\right)^{1 / 2}=\frac{\alpha}{\sqrt{3 \eta}}=\left(\frac{\alpha}{3 \psi^{\prime}(\alpha)}\right)^{1 / 2}$.

For $\alpha \rightarrow 0$ (perfect gas), $\mathcal{N} \sim 1$ and for $\alpha \rightarrow+\infty$ (singular sphere), $\mathcal{N} \sim \frac{\alpha}{\sqrt{6}} \rightarrow+\infty$. Therefore the number $\mathcal{N}$ quantifies the inhomogeneity of the self-gravitating gas. The evolution of $\mathcal{N}(\alpha)$ along the spiral is ploted in Fig. 14. From Eq. (105), $\mathcal{N}$ is just the ratio between the central density and the average density to the power $1 / 2$.

\section{Conclusion}

In the first part of the paper, we have investigated the stability of isothermal spheres in the canonical ensemble using a thermodynamical approach. This is a natural extension of the work of Padmanabhan (1989) in 
the microcanonical ensemble. As is well known, there is no hydrostatic equilibrium for an isothermal gas below a critical temperature $k T_{\mathrm{c}}=\frac{G m M}{2.52 R}$ (Lynden-Bell \& Wood 1968). This corresponds to a situation in which the domain size becomes comparable with the Jeans length, i.e. $R>0.916 \ldots L_{\mathrm{J}}$. In that case, we expect the system to collapse. By analyzing the second variations of free energy, we have found that the point of minimum temperature is precisely the point at which the free energy ceases to be a local maximum and becomes a saddle point in the series of equilibrium. This vindicates the theorem of Katz (1978) and provides in addition the form of the perturbation that induces instability. Contrary to the microcanonical ensemble, it has not a "core-halo" structure, nor multiple oscillations. We expect therefore the system to collapse without fragmenting. On the other hand, if we start from isothermal gaseous spheres with high density contrasts $\mathcal{R}>32.1$ (this corresponds to $R>2.996 \ldots L_{\mathrm{K}}$ ), such spheres are unstable saddle points and probably fragment into small "clumps" (although they would be said to be stable by a naive Jeans argument since $\left.R<0.916 \ldots L_{\mathrm{J}}\right)$. The number of clumps, presumably associated with the number of zeros in the mode of instability, increases with the density contrast or, equivalently, as the domain size becomes larger and larger with respect to the King's radius. The distinction between the Jeans length and the King's length is essential in the present context and had not been noticed previously. The Jeans length is related to the parameter $\eta$ determining whether an hydrostatic equilibrium state exists or not for an isothermal sphere while the King's length is related to the parameter $\alpha$ parametrizing the series of equilibrium. These are very different parameters.

In the second part of the paper, we have extended the Jeans instability criterion to the case of inhomogeneous bounded gaseous spheres described by NavierStokes equations. This treatment avoids the well-known difficulties associated with an infinite and homogeneous medium (Jeans swindle). Quite remarkably, we have provided an elegant analytical solution of this more general problem without making any approximation.

Now, the question that naturally emerges is what happens to a gravitationally unstable gaseous configuration. The collapse of isothermal gas spheres has been investigated by Penston (1969) using Navier-Stokes equations (without viscosity). He found an analytical solution for which the collapse is self-similar and develops a finite time singularity (i.e., the central density becomes infinite in a finite time). This only partially answers the above question. Indeed, his solution is just a particular solution of the fluid equations and more general solutions, which are not necessarily spherically symmetric, may describe a fragmentation process. In addition, Penston (1969) works in an infinite domain for which the isothermal spheres have infinite mass and are always unstable. Therefore, he could not evidence a phase transition between an equilibrium "gaseous" state and a "collapsed" state, depending on the value of the temperature.
The thermodynamics of self-gravitating systems is so intriguing that we have been tempted to explore this problem anew from a dynamical point of view (Chavanis et al. 2001). We have introduced a numerical algorithm in the form of a relaxation equation (Chavanis 1996, 2001a; Chavanis et al. 1996) constructed so as to increase monotonically entropy at fixed energy in the microcanonical ensemble and free energy at fixed temperature in the canonical ensemble. With this numerical algorithm, we can cover the whole bifurcation diagram in parameter space and check, by an independant method, the stability limits of Katz (1978). We have verified that the density profile that induces instability at the critical point has a "corehalo" structure in the microcanonical ensemble but not in the canonical ensemble, in agreement with the study of Padmanabhan (1989) and the present study. We have also checked that the number of oscillations in the perturbation profile increases when we start from unstable isothermal spheres with high density contrasts. With this algorithm, we have explored the "bassin of attraction" of the stable isothermal spheres. Since they only are local maxima of entropy or free energy, an initial condition can either relax towards such a metastable equilibrium state or collapse, depending on its topology. Hence, the control parameter $\Lambda$ or $\eta$ is not sufficient to characterize completely the final state of the system even in the case when an equilibrium exists. This depends whether the initial condition lies in the "bassin of attraction" of the equilibrium solution or not. The complete characterization of this bassin of attraction is difficult but we have given some examples. It is also possible to include rotation in our numerical algorithm in order to compute entropy maxima that are not spherically symmetric and determine the influence of angular momentum conservation on the collapse of a rotating self-gravitating system (Chavanis \& Rosier, in preparation). Some interesting works have been done in that direction (Lagoute \& Longaretti 1996; Laliena 1999; Fliegans \& Gross 2001; Lynden-Bell 2000) but the complete description of rotating isothermal gaseous spheres is still in its infancy stage.

It is also of first interest to test numerically the speculations that we have made concerning the development of the instability, i.e. if the system will collapse as a whole or if it will fragment and break into substructures. These two regimes are clearly separated, at least in the theoretical model that we have considered here (Antonov model). We have suggested that if we start from a solution of the Emden equation with a high density contrast, it should break in a series of "clumps" associated with the oscillations of the density perturbation that we have calculated in the linear regime. Obviously, if we want to follow the nonlinear evolution correctly, and see the fragmentation, we must relax the hypothesis of spherical symmetry.

In this paper, we have assumed that the particles can be treated by classical mechanics and that they are point-like. The extension of our work to general relativity (for, e.g., neutron stars) is almost straightforward (Chavanis 2001b). We find that there is no hydrostatic 
equilibrium if the system size is smaller than a multiple of the Schwarzschild radius. We also show analytically that the point of smallest radius (for a given mass $M)$ corresponds precisely to the point at which the series of equilibrium becomes unstable. Secondary modes of instability are found; they follow a geometric progression like in the Newtonian case but with a different ratio. When the speed of light goes to $+\infty$, we recover the results of the present study. It is seen that relativistic effects favour gravitational instability, i.e. instability occurs sooner than in the Newtonian limit. On the other hand, the case of self-gravitating fermions enclosed within a box has been considered by Chavanis \& Sommeria (1998). This study was formulated in the context of the "violent relaxation" of collisionless stellar systems introduced by Lynden-Bell (1967) but the results also apply to quantum particles such as massive neutrinos in Dark Matter models (Ingrosso et al. 1992) or degenerate electrons in White Dwarf stars (Chandrasekhar 1942). When degeneracy is accounted for, there exists a global entropy maximum for all values of energy (Chavanis \& Sommeria 1998; Robert 1998). It has a "core-halo" structure with a degenerate core and a dilute atmosphere. Depending on the degeneracy parameter, there can be a "gravothermal catastrophe" at $E=-0.335 G M^{2} / R$ but the core ceases to shrink when it becomes degenerate. Considering a classical gas with a short distance cut-off $a$ essentially leads to the same results (see, e.g., Padmanabhan 1990; Follana \& Laliena 2000).

For astrophysical purposes, it is still a matter of debate to decide whether collisionless stellar systems like elliptical galaxies are degenerate (in the sense of Lynden-Bell) or not. Since degeneracy can stabilize the system without changing its overall structure at large radii, we have suggested that degeneracy could play a role in galactic nuclei (Chavanis \& Sommeria 1998). The recent simulations of Leeuwin \& Athanassoula (2000) and the theoretical model of Stiavelli (1998) seem to go in that direction: if the nucleus of elliptical galaxies contains a (primordial) black hole, degeneracy must be taken into account and can explain the cusps observed in the center of galaxies. This form of degeneracy is also relevant for massive neutrinos in Dark Matter models where it competes with quantum degeneracy (Kull et al. 1996).

All together, these results suggest that the statistical mechanics and the thermodynamics of self-gravitating systems can have quite relevant astrophysical applications at different scales of the universe: stars (Chandraskhar 1942), interstellar medium (de Vega et al. 1996a, 1996b), globular clusters (Lynden-Bell \& Wood 1968), elliptical galaxies (Lynden-Bell 1967; Hjorth \& Madsen 1993; Chavanis \& Sommeria 1998), dark matter (Ingrosso et al. 1992; Kull et al. 1996), cosmology (Saslaw \& Hamilton 1984; de Vega et al. 1998)... The same ideas of statistical mechanics have been introduced in two-dimensional turbulence to explain the formation and maintenance of coherent vortices, like Jupiter's Great Red Spot, which are common features of large-scale geophysical and astrophysical flows (see Bouchet \& Sommeria 2000; Bouchet et al. 2001 and references therein). The analogy between the statistical mechanics of two-dimensional vortices and stellar systems has been described in Chavanis (1996, 1998b, 2001c). This analogy concerns not only the equilibrium state (the formation of large-scale structures) but also the relaxation towards equilibrium (Chavanis et al. 1996; Chavanis 2001d) and the statistics of fluctuations (Chavanis \& Sire 2000). These ideas may also have applications in the context of planet formation where large-scale vortices present in the solar nebula could efficiently trap dust particles to form the planetesimals and the planets (Barge \& Sommeria 1995; Tanga et al. 1996; Bracco et al. 1999; Chavanis 2000). Accordingly, the statistical mechanics of these nonlinear media seems to be able to account for the fascinating process of self-organization in nature.

Acknowledgements. I acknowledge interesting discussions with L. Athanassoula, F. Combes, O. Fliegans, J. Hjorth, H. Kandrup, V. Laliena, F. Leeuwin, D. Lynden-Bell, T. Padmanabhan, R. Robert, B. Semelin and J. Sommeria on these exciting problems of thermodynamics. This work was initiated during my stay at the Institute for Theoretical Physics, Santa Barbara, during the program on Hydrodynamical and Astrophysical Turbulence (February-June 2000). This research was supported in part by the National Science Foundation under Grant No. PHY94-07194.

\section{References}

Antonov, V. A. 1962, Vest. Leningr. Gos. Univ., 7, 135

Barge, P., \& Sommeria, J. 1995, A\&A, 295, L1

Bouchet, F., \& Sommeria, J. 2000, submitted to J. Fluid. Mech. [physics/0003079]

Bouchet, F., Chavanis, P. H., \& Sommeria, J., in preparation Binney, J., \& Tremaine, S. 1987, Galactic Dynamics (Princeton Series in Astrophysics)

Bracco, A., Chavanis, P. H., Provenzale, A., \& Spiegel, E. A. 1999, Phys. Fluids, 11, 2280

Cerruti-Sola, M., Cipriani, P., \& Pettini, M. 2001, MNRAS, submitted [astro-ph/0105380]

Chandrasekhar, S. 1942, An Introduction to the Theory of Stellar Structure (Dover)

Chavanis, P. H. 1996, Ph.D. Thesis, École Normale Supérieure de Lyon

Chavanis, P. H. 1998a, MNRAS, 300, 981

Chavanis, P. H. 1998b, Annals N.Y. Acad. Sci., 867, 120

Chavanis, P. H. 2000, A\&A, 356, 1089

Chavanis, P. H. 2001a, Statistical mechanics of violent relaxation in stellar systems, in: Proc. of the Conference on Multiscale Problems in Science and Technology (Springer) Chavanis, P. H. 2001b, to appear in A\&A [astro-ph/0108230]

Chavanis, P. H. 2001c, On the analogy between twodimensional vortices and stellar systems, in Proc. of the IUTAM Symp. on Geometry and Statistics of Turbulence, ed. T. Kambe, T. Nakano, \& T. Miyauchi (Kluwer Academic Publishers)

Chavanis, P. H. 2001d, Phys. Rev. E, 64, 026309

Chavanis, P. H., Rosier, C., \& Sire, C. 2001, Phys. Rev. E, submitted [cond-mat/0107345]

Chavanis, P. H., \& Sire, C. 2000, Phys. Rev. E, 62, 490

Chavanis, P. H., \& Sommeria, J. 1998, MNRAS, 296, 569 
Chavanis, P. H., Sommeria, J., \& Robert, R. 1996, ApJ, 471, 385

Cohn, H. 1980, ApJ, 242, 765

de Vega, H. J., Sanchez, N., \& Combes, F. 1996a, Nature, 383, 56

de Vega, H. J., Sanchez, N., \& Combes, F. 1996b, Phys. Rev. $\mathrm{D}, 54,6008$

de Vega, H. J., Sanchez, N., \& Combes, F. 1998, ApJ, 500, 8

de Vega, H. J., \& Sanchez, N. 2001a [astro-ph/0101568]

de Vega, H. J., \& Sanchez, N. 2001b [astro-ph/0101567]

Dubrulle, B., \& Graner, F. 1994, A\&A, 282, 269

Fliegans, O., \& Gross, D. H. E. 2001, Phys. Rev. E., submitted [cond-mat/0102062]

Follana, E., \& Laliena, V. 2000, Phys. Rev. E, 61, 6270

Graner, F., \& Dubrulle, B. 1994, A\&A, 282, 262

Hjorth, J., \& Madsen, J., 1993, MNRAS, 265, 237

Ingrosso, G., Merafina, M., Ruffini, R., \& Strafella, F. 1992, A\&A, 258, 223

Katz, J. 1978, MNRAS, 183, 765

Kull, A., Treumann, R. A., \& Böringer, H. 1996, ApJ, 466, L1

Lagoute, C., \& Longaretti, P. Y. 1996, A\&A, 308, 441

Laliena, V. 1999, Phys. Rev. E, 59, 4786

Larson, R. B. 1970, MNRAS, 147, 323

Leeuwin, F., \& Athanassoula, E. 2000, MNRAS, 417, 79
Lynden-Bell, D. 1967, MNRAS, 136, 101

Lynden-Bell, D. 2000 [astro-ph/0007116]

Lynden-Bell, D., \& Eggleton, P. P. 1980, MNRAS, 191, 483

Lynden-Bell, D., \& Lynden-Bell, R. M. 1977, MNRAS, 181, 405

Lynden-Bell, D., \& Wood, R. 1968, MNRAS, 138, 495

Ogorodnikov, K. F. 1965, Dynamics of stellar systems (Pergamon)

Padmanabhan, T. 1989, ApJS, 71, 651

Padmanabhan, T. 1990, Phys. Rep., 188, 285

Penston, M. V. 1969, MNRAS, 144, 425

Pfenniger, D., Combes, F., \& Martinet, L. 1994, A\&A, 285, 79

Pfenniger, D., \& Combes, F. 1994, A\&A, 285, 94

Robert, R. 1998, Class. Quantum Grav., 15, 3827

Saslaw, W. C., \& Hamilton, A. J. S. 1984, ApJ, 276, 13

Semelin, B., de Vega, H. J., Sanchez, N., \& Combes, F. 1999, Phys. Rev. D, 59, 125021

Semelin, B., Sanchez, N., de Vega, H. J. 2001, Phys. Rev. D, 63, 084005

Shapiro, S. L., \& Teukolsky, S. A. 1995, ApJ, 292, L41

Stiavelli, M., 1998, ApJ, 495, L91

Tanga, P., Babiano, A., Dubrulle, B., \& Provenzale, A. 1996, Icarus, 121, 158

Yabushita, S. 1968, MNRAS, 140, 109 